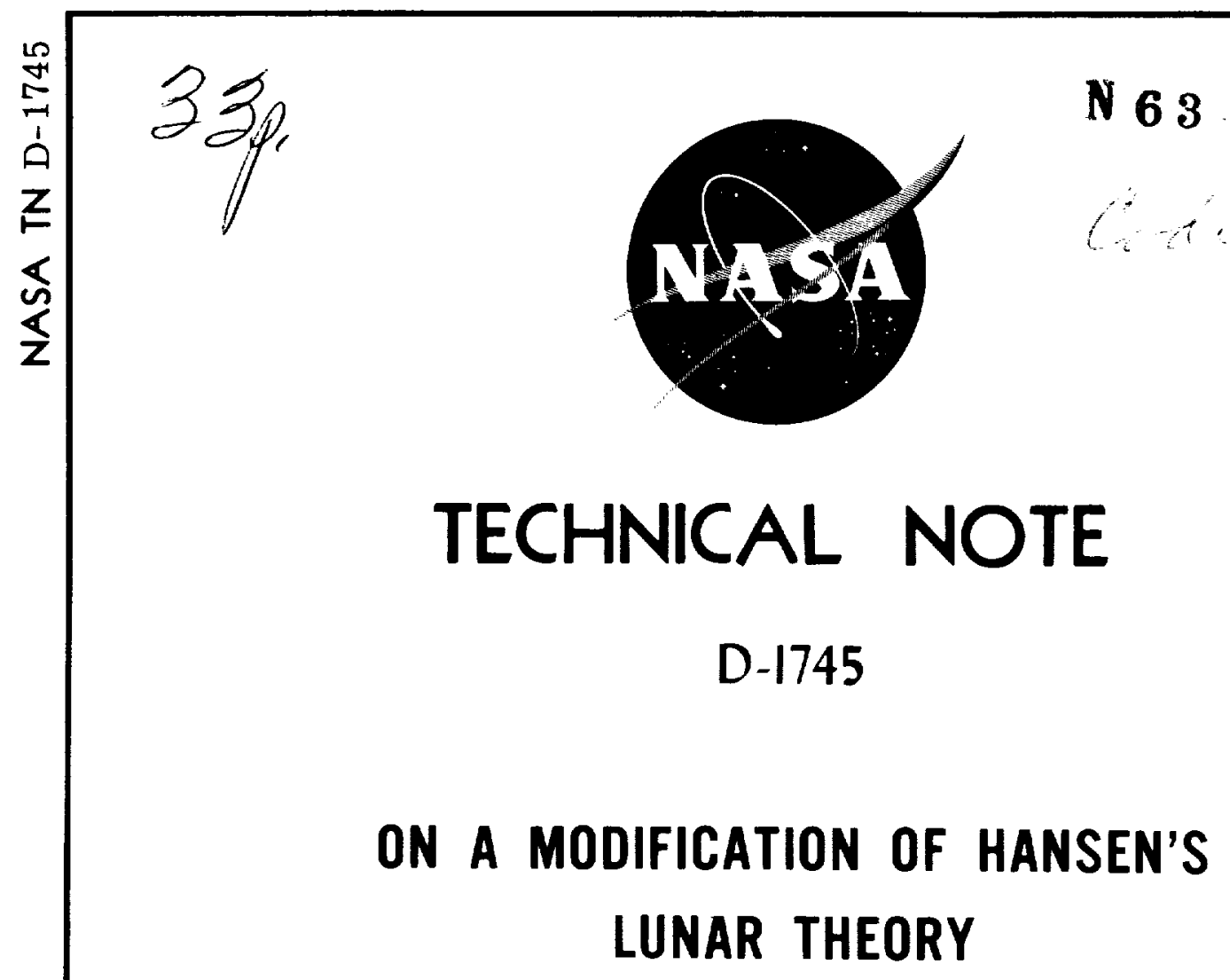

\author{
Peter Musen
}

Goddard Space Flight Center

Greenbelt, Maryland

NATIONAL AERONAUTICS AND SPACE ADMINISTRATION WASHINGTON

June 1963 
a 


\title{
ON A MODIFICATION OF HANSEN'S LUNAR THEORY
}

\author{
by \\ Peter Musen \\ Goddard Space Flight Center
}

SUMMARY

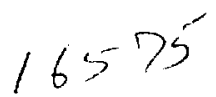

A modification of Hansen's lunar theory is given in a form that permits a purely numerical treatment of solar perturbations of planetary satellites or of perturbations caused by the earth in the motion of hypothetical lunar satellites. The development of the coordinates and of the velocities of a satellite is obtained in the form of a trigonometric series in four arguments with numerical coefficients. At the earlier stages of the computation we are not compelled to use Hansen's basic w-function: We make use of the fictitious mean anomaly and the replacement "bar" operation - two characteristic features of Hansen's theory - to set up a process of iteration in a convenient form, rather than to compute $w$. The preference given to the method of iteration was motivated by the circumstance that input information must not necessarily be limited to the choice of the rotating ellipse as an intermediary orbit. A systematic investigation of the orbital stability of lunar satellites can be based on the development given herein. 



\section{CONTENTS}

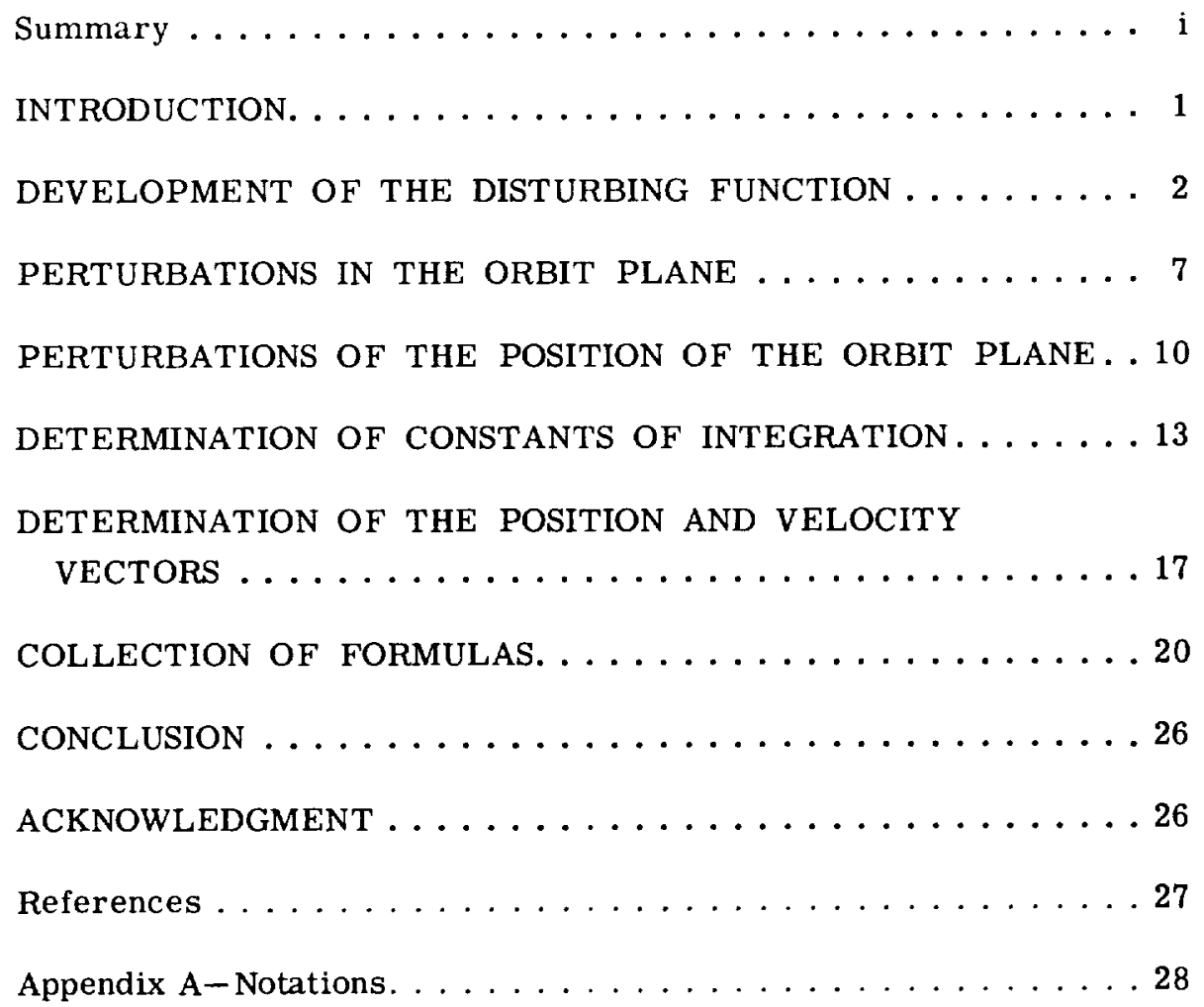





\title{
ON A MODIFICATION OF HANSEN'S LUNAR THEORY*
}

\author{
by \\ Peter Musen \\ Goddard Space Flight Center
}

\section{INTRODUCTION}

In this paper a modification of Hansen's lunar theory (Reference 1) is given in a form that permits a purely numerical treatment of solar perturbations of planetary satellites as well as the perturbations caused by the earth in the motion of hypothetical lunar satellites. The development of perturbations of very close lunar satellites does not present any difficulty and therefore is not given here. The case of more distant satellites was treated by Lemekhova (Reference 2) on the basis of Delaunay's theory for orbits with low inclinations toward the orbit of the moon. At the present time we are not in possession of a complete analytical theory of satellites with highly inclined orbits, although Delaunay's theory can be modified to become valid for such orbits. Because of this circumstance, the use of a semi-analytical theory is suggested, as given in this paper.

Hansen's original lunar theory requires the use of the derivatives of the disturbing functions with respect to the eccentricity and the parameters determining the orbit plane's position. This means that a literal development of the disturbing function must be obtained before the numerical values of the elements can be substituted. However, with the advent of electronic machines, more emphasis can be put on the development of purely numerical theories.

Unlike Hansen's lunar theory, his planetary theory (Reference 3) is a purely numerical one. For this reason the theory of perturbations in the orbit plane, as presented herein, takes Hansen's planetary theory, and not his lunar theory, as a pattern. In addition, at the earlier stages of the computations we are not compelled to use Hansen's basic $w$-function. Instead, we make use of the fictitious mean anomaly and the replacement "bar" operation to set up the process of iteration in a convenient for $m$, rather than to compute $w$. The preference given to the method of iteration over the method of computing perturbations of different orders was motivated by the circumstance that input information must not necessarily be limited to the choice of Hansen's rotating ellipse as an intermediary orbit. In addition, the programming and the computational process become more uniform if the method of iteration is used.

* Also to be published in the Journal of Geophysical Research. 
Only after the process of iteration is completed is the $\bar{W}$-function formed in order to determine the perturbations in the mean anomaly and in the radius vector.

The orbit of the disturbing body is taken to be a moving ellipse.

The inclination of the satellite's orbit plane toward the orbit plane of the disturbing body can be large. For this reason we do not deduce the standard development of the latitude into series, but make use of four "redundant" parameters introduced by the author in the theory of artificial satellites (Reference 4). The introduction of these parameters makes all basic arguments linear in time from the outset, and it permits us to deduce the rectangular components of the position and velocity vectors very easily.

The knowledge of these components facilitates the computation of osculating elements.

To simplify the form of the development, we take - as did Hansen - the orbit plane of the disturbing body as a basic reference plane.

The effect of this plane's motion on the relative motion of the satellite is very small and can easily be taken into consideration.

The form of the disturbing function in our case favors only the choice of time as an independent variable, but not the true or the eccentric anomaly as in the case of an artificial satellite of the earth.

\section{DEVELOPMENT OF THE DISTURBING FUNCTION}

For a satellite of negligible mass, we have

$$
\begin{aligned}
\Omega= & \frac{m^{\prime} r^{2}}{r^{\prime 3}}\left(\frac{3}{2} S^{2}-\frac{1}{2}\right) \\
& +\frac{m^{\prime} r^{3}}{r^{\prime 4}}\left(\frac{5}{2} S^{3}-\frac{3}{2} S\right) \\
& +\frac{m^{\prime} r^{4}}{r^{-5}}\left(\frac{35}{8} S^{4}-\frac{15}{4} S^{2}+\frac{3}{8}\right)+\cdots .
\end{aligned}
$$

(See Appendix A for list of notations.) Putting

$$
\begin{aligned}
& s=\frac{r}{a} \cdot \frac{a^{\prime}}{r^{\prime}} s, \\
& p=\frac{r}{a} \cdot \frac{a^{\prime}}{r^{\prime}},
\end{aligned}
$$


we deduce:

$$
\begin{aligned}
\Omega= & \frac{m^{\prime} a^{2}}{a^{3}} \cdot \frac{a^{\prime}}{r^{\prime}} \cdot\left(\frac{3}{2} s^{2}-\frac{1}{2} p^{2}\right) \\
& +\frac{m^{\prime} a^{3}}{a^{\prime 4}} \cdot \frac{a^{\prime}}{r^{\prime}} \cdot\left(\frac{5}{2} s^{3}-\frac{3}{2} p^{2} s\right) \\
& +\frac{m^{\prime} a^{4}}{a^{\prime 5}} \cdot \frac{a^{\prime}}{r^{\prime}}\left(\frac{35}{8} s^{4}-\frac{15}{4} s^{2} p^{2}+\frac{3}{8} p^{4}\right)+\ldots, \\
r \frac{\partial \Omega}{\partial r}=a \frac{\partial \Omega}{\partial a}= & 2 \frac{m^{\prime} a^{2}}{a^{\prime 3}} \cdot \frac{a^{\prime}}{r^{\prime}} \cdot\left(\frac{3}{2} s^{2}-\frac{1}{2} p^{2}\right) \\
& +3 \frac{m^{\prime} a^{3}}{a^{\prime 4}} \cdot \frac{a^{\prime}}{r^{7}}\left(\frac{5}{2} s^{3}-\frac{3}{2} p^{2} s\right) \\
& +4 \frac{m^{\prime} a^{4}}{a^{\prime 5}} \cdot \frac{a^{\prime}}{r^{\prime}} \cdot\left(\frac{35}{8} s^{4}-\frac{15}{4} s^{2} p^{2}+\frac{3}{8} p^{4}\right)+\ldots,
\end{aligned}
$$

and

$$
\begin{aligned}
\frac{a}{r} \cdot \frac{r^{\prime}}{a^{\prime}} \frac{\partial \Omega}{\partial S}= & 3 \frac{m^{\prime} a^{2}}{a^{\prime 3}} \cdot \frac{a^{\prime}}{r^{\prime} s} \\
& +\frac{m^{\prime} a^{3}}{a^{14}} \cdot \frac{a^{\prime}}{r^{\prime}}\left(\frac{15}{2} s^{2}-\frac{3}{2} p^{2}\right) \\
& +\frac{m^{\prime} a^{4}}{a^{\prime 5}} \cdot \frac{a^{\prime}}{r^{\prime}}\left(\frac{35}{2} s^{3}-\frac{15}{2} p^{2} s\right) .
\end{aligned}
$$

We have

$$
\begin{gathered}
\mathrm{S}=\cos (\mathrm{v}-\psi) \cos \left(\mathrm{v}^{\prime}-\psi^{\prime}\right)+\sin (\mathrm{v}-\psi) \sin \left(\mathrm{v}^{\prime}-\psi^{\prime}\right) \cos \mathrm{I}, \\
\mathbf{s}=\frac{\mathrm{r}}{\mathrm{a}} \cdot \frac{\mathrm{a}^{\prime}}{\mathbf{r}^{\prime}} \cos (\mathrm{v}-\psi) \cos \left(\mathrm{v}^{\prime}-\psi^{\prime}\right) \\
\quad+\frac{\mathbf{r}}{\mathrm{a}} \cdot \frac{\mathrm{a}^{\prime}}{\mathbf{r}^{\prime}} \sin (\mathrm{v}-\psi) \sin \left(\mathrm{v}^{\prime}-\psi^{\prime}\right) \cos \mathrm{I} .
\end{gathered}
$$

Taking the relations

$$
\begin{aligned}
& 2 \mathrm{~N}=\psi_{0}+\psi_{0}^{\prime}-\psi-\psi^{\prime}-2 \mathrm{n}_{0} a \mathrm{t}, \\
& 2 \mathrm{~K}=\psi_{0}-\psi_{0}^{\prime}-\psi+\psi^{\prime}+2 \mathrm{n}_{0} \eta \mathrm{t},
\end{aligned}
$$




$$
\begin{aligned}
v & =\bar{f}+\pi_{0}+n_{0} y t, \\
v^{\prime} & =\bar{f}^{\prime}+\pi_{0}^{\prime}+n_{0} y^{\prime} t
\end{aligned}
$$

into account, we deduce from the Equations 6 and $6^{\prime}$ that

$$
\begin{gathered}
S=+\left(\lambda_{1}^{2}-\lambda_{2}^{2}\right) \cos \left(\overline{\mathrm{f}}+\overline{\mathrm{f}}^{\prime}+\omega+\omega^{\prime}\right)-2 \lambda_{1} \lambda_{2} \sin \left(\overline{\mathrm{f}}+\overline{\mathrm{f}}^{\prime}+\omega+\omega^{\prime}\right) \\
+\left(\lambda_{4}^{2}-\lambda_{3}^{2}\right) \cos \left(\overline{\mathrm{f}}-\overline{\mathrm{f}}^{\prime}+\omega-\omega^{\prime}\right)-2 \lambda_{3} \lambda_{4} \sin \left(\overline{\mathrm{f}}-\overline{\mathrm{f}}^{\prime}+\omega-\omega^{\prime}\right) \\
\mathrm{s}=(1+\nu)\left[+\left(\lambda_{1}^{2}-\lambda_{2}{ }^{2}\right) \frac{\overline{\mathrm{r}}}{\mathrm{a}} \cdot \frac{\mathrm{a}^{\prime}}{\overline{\mathrm{r}}^{\prime}} \cos \left(\overline{\mathrm{f}}+\overline{\mathrm{f}}^{\prime}+\omega+\omega^{\prime}\right)\right. \\
-2 \lambda_{1} \lambda_{2} \frac{\overline{\mathrm{r}}}{\mathrm{a}} \cdot \frac{\mathrm{a}^{\prime}}{\overline{\mathrm{r}}^{\prime}} \sin \left(\overline{\mathrm{f}}+\overline{\mathrm{f}}^{\prime}+\omega+\omega^{\prime}\right) \\
+\left(\lambda_{4}{ }^{2}-\lambda_{3}{ }^{2}\right) \frac{\overline{\mathrm{r}}_{\mathrm{a}}}{\mathrm{a}^{\prime}} \cdot \frac{\mathrm{a}^{\prime}}{\overline{\mathrm{r}}^{\prime}} \cos \left(\overline{\mathrm{f}}-\overline{\mathrm{f}}^{\prime}+\omega-\omega^{\prime}\right) \\
\left.-2 \lambda_{3} \lambda_{4} \frac{\overline{\mathrm{r}}}{\mathrm{a}} \cdot \frac{\mathrm{a}^{\prime}}{\overline{\mathrm{r}}^{\prime}} \sin \left(\overline{\mathrm{f}}-\overline{\mathrm{f}}^{\prime}+\omega-\omega^{\prime}\right)\right]
\end{gathered}
$$

where

$$
\begin{aligned}
& \omega=\omega_{0}+n_{0}(y+a-\eta) t, \\
& \omega_{0}=\pi_{0}-\psi_{0}, \\
& \omega^{\prime}=\omega_{0}^{\prime}+n_{0}\left(y^{\prime}+a+\eta\right) t, \\
& \omega_{0}^{\prime}=\pi_{0}^{\prime}-\psi_{0}^{\prime} .
\end{aligned}
$$

The arguments $\omega$ and $\omega^{\prime}$ are defined as linear functions of time from the very start.

The next step is to obtain the developments of

$$
\frac{\bar{r}}{a} \cdot \frac{a^{\prime}}{\bar{r}} \cos \sin \left(\bar{f} \pm \bar{f}^{\prime}+\omega \pm \omega^{\prime}\right)
$$

and

$$
\frac{\bar{r}}{a} \cdot \frac{a^{\prime}}{\bar{r}^{\prime}}
$$

These five series represent an input. They can be easily computed, even using a desk calculator, by means of Cayley's scheme (Reference 5). We have: 


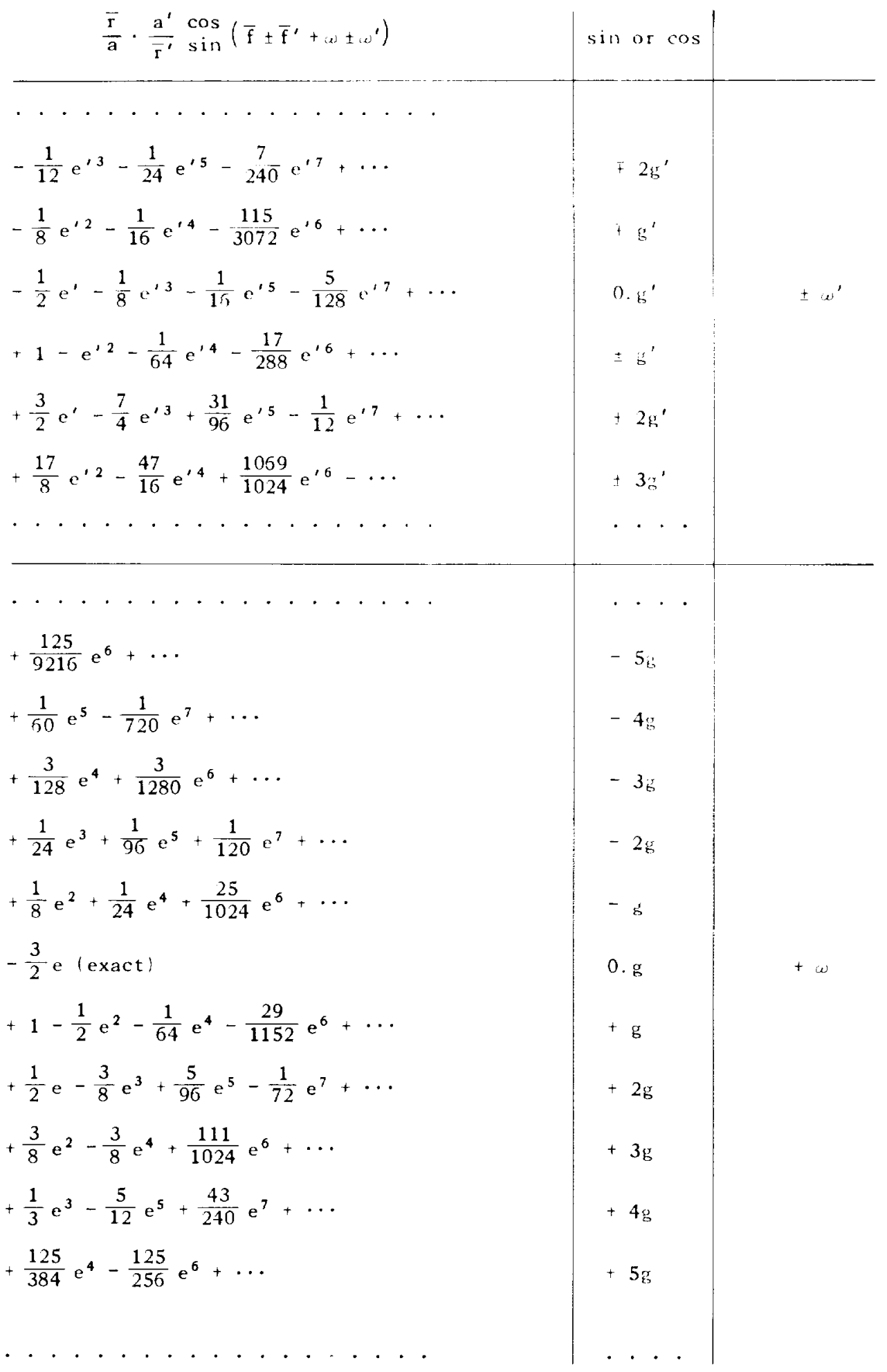


In this scheme we multiply every line of the upper part by every line of the lower part and add the arguments together, then attach the sin or cos symbol to each term of the product. The upper sign of the argument in the solar part is associated with the development of

$$
\frac{\bar{r}}{a} \cdot \frac{a^{\prime}}{\bar{r}^{\prime}} \cos \sin \left(\bar{f}+\bar{f}^{\prime}+\omega+\omega^{\prime}\right)
$$

and the lower sign is associated with the development of

$$
\overline{\mathbf{r}} \cdot \frac{a^{\prime}}{\bar{r}} \cos \sin \left(\bar{f}-\bar{f}^{\prime}+\omega-\omega^{\prime}\right)
$$

The numerical values of $e$ and $\mathrm{e}^{\prime}$ can be substituted from the very start.

In the same manner we have:

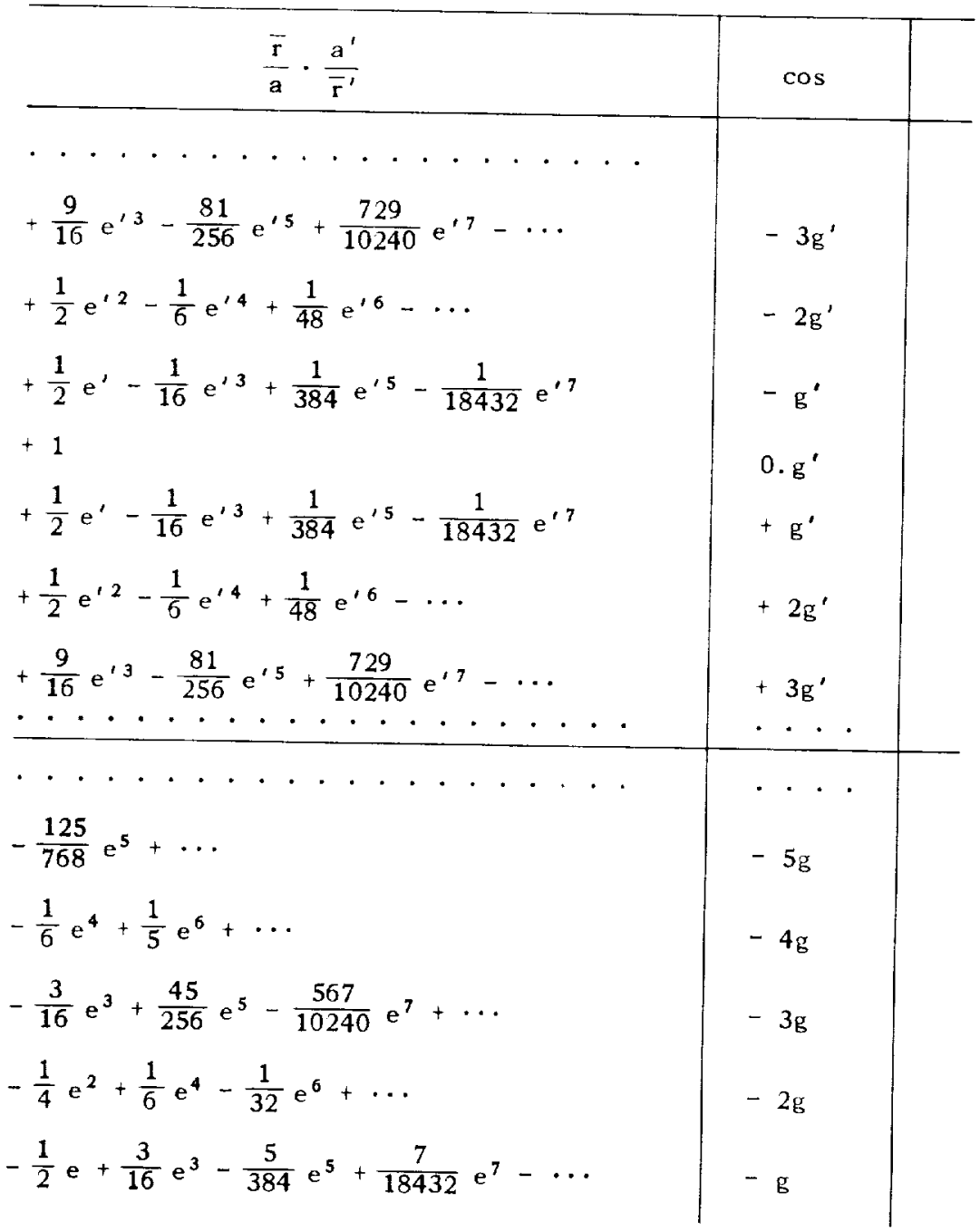




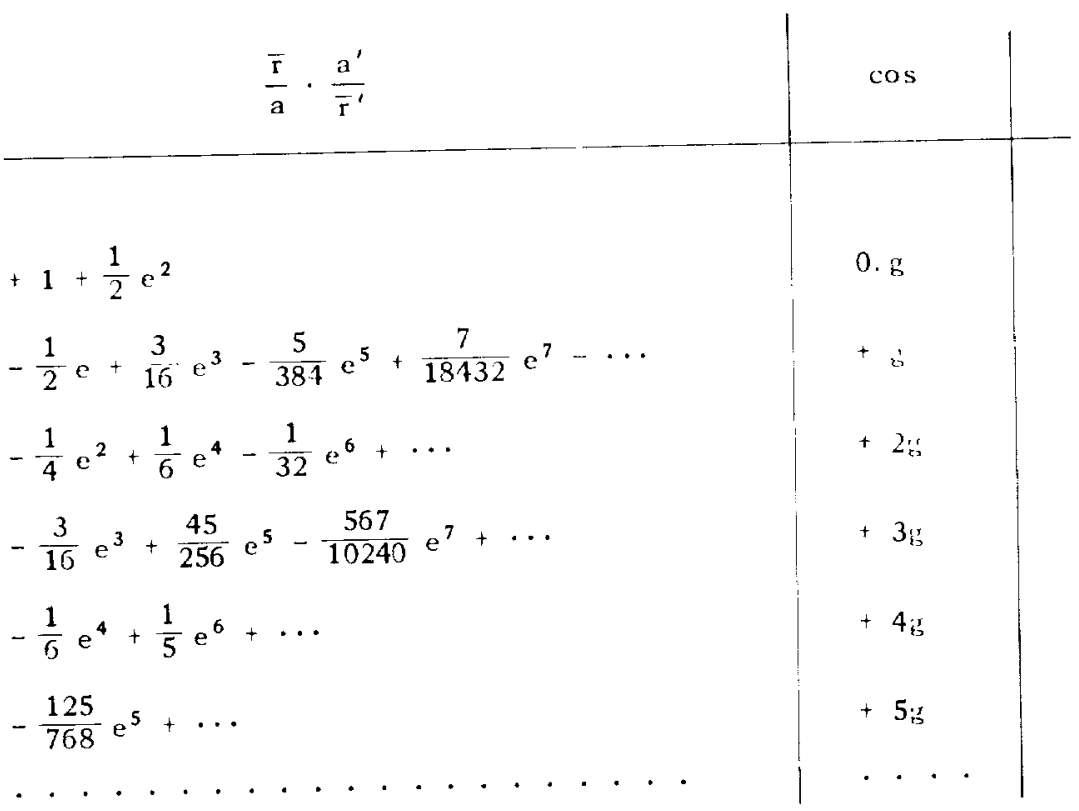

\section{PERTURBATIONS IN THE ORBIT PLANE}

To determine the perturbations in the radius vector and in the mean anomaly, Hansen from the very start introduces the function

$$
W=\Xi+\Upsilon\left(\frac{\rho}{a_{0}} \cos \varphi+\frac{3}{2} e_{0}\right)+\psi \cdot \frac{\rho}{a_{0}} \sin \varphi
$$

where

$$
\begin{gathered}
\Xi=-1-\frac{h_{0}}{h}+2 \frac{h}{h_{0}}-3 e_{0} \frac{h}{h_{0}} \cdot \frac{e \cos \left(x-n_{0} y t-\pi_{0}\right)-e_{0}}{1-e_{0}^{2}} . \\
\Upsilon=2 \frac{h}{h_{0}} \cdot \frac{e \cos \left(x-n_{0} y t-\pi_{0}\right)-e_{0}}{1-e_{0}^{2}} . \\
\Psi=2 \frac{h}{h_{0}} \cdot \frac{e \sin \left(x-n_{0} y t-\pi_{0}\right)}{1-e_{0}^{2}} .
\end{gathered}
$$

From the standpoint of the process of iteration the use of the elements

$$
\frac{h_{0}}{i}, \quad r \quad \Psi
$$


would be preferable. We have (Reference 1)

$$
\begin{gathered}
\frac{d}{d t} \frac{h_{0}}{h}=h_{0} \frac{\partial \Omega}{\partial f} \\
\frac{d r}{d t}=n_{0} y+\frac{2 a_{0} n_{0}}{\sqrt{1-e_{0}^{2}}}\left\{\left[\left(\frac{a_{0}}{r}+\frac{h^{2}}{h_{0}^{2}} \cdot \frac{1}{1-e_{0}^{2}}\right) \cos \bar{f}+\frac{e_{0}}{1-e_{0}^{2}} \frac{h^{2}}{h_{0}^{2}}\right] \frac{\partial \Omega}{\partial f}+\frac{a_{0}}{r} \sin \bar{f}\left(r \frac{\partial \Omega}{\partial r}\right)\right\} \\
\frac{d \Psi}{d t}=-n_{0} y\left(r+2 \frac{h}{h_{0}} \cdot \frac{e_{0}}{1-e_{0}^{2}}\right)+\frac{2 a_{0} n_{0}}{\sqrt{1-e_{0}^{2}}}\left\{\left(\frac{a_{0}}{r}+\frac{h^{2}}{h_{0}^{2}} \cdot \frac{1}{1-e_{0}^{2}}\right) \sin \bar{f} \frac{\partial \Omega}{\partial f}-\frac{a_{0}}{r} \cos \bar{f} \cdot\left(r \frac{\partial \Omega}{\partial r}\right)\right\} \cdot(16)
\end{gathered}
$$

Taking into account

$$
\frac{\partial \Omega}{\partial \mathrm{f}}=\frac{1}{\sqrt{1-\mathrm{e}_{0}^{2}}}\left(\frac{\overline{\mathrm{r}}^{2}}{\mathrm{a}_{0}^{2}} \frac{\partial \Omega}{\partial l}-\frac{\overline{\mathrm{r}}}{\mathrm{a}_{0}} \cdot \frac{\mathrm{e}_{0} \sin \overline{\mathrm{f}}}{\sqrt{1-\mathrm{e}_{0}^{2}}} \cdot \mathrm{r} \frac{\partial \Omega}{\partial \mathrm{r}}\right)
$$

we transform Equation 15 to the form

$$
\frac{\mathrm{d} \Upsilon}{\mathrm{dt}}=+\mathrm{n}_{0} \mathrm{y} \Psi+\mathrm{m}_{1} \frac{\partial \Omega}{\partial t}+N_{1} \cdot \mathbf{r} \frac{\partial \Omega}{\partial \mathrm{r}}
$$

where

$$
i_{1}=\frac{2 a_{0} n_{0}}{1-e_{0}^{2}}\left[\frac{1}{e_{0}}\left(1-e_{0}^{2}-\frac{\bar{r}^{2}}{a_{0}^{2}}\right)-\frac{\nu}{1+\nu} \frac{1}{e_{0}}\left(1-e_{0}^{2}-\frac{\bar{r}}{a_{0}}\right)+\left(\frac{h^{2}}{h_{0}^{2}}-1\right) \frac{1}{e_{0}} \frac{\bar{r}}{a_{0}}\left(1-\frac{\bar{r}}{a_{0}}\right)\right]
$$

and

$$
N_{1}=\frac{2 a_{0} n_{0}}{1-e_{0}^{2}} \cdot \frac{\bar{r}}{a_{0}} \frac{\sin \bar{f}}{\sqrt{1-e_{0}^{2}}}\left[1-\frac{a_{0}}{\bar{r}} \cdot \frac{\nu}{1+\nu}-\left(\frac{h^{2}}{h_{0}^{2}}-1\right)\left(\frac{a_{0}}{\bar{r}}-1\right)\right] .
$$

In a similar manner, using the basic relations

$$
\begin{aligned}
& \frac{\bar{r}}{a_{0}} \sin \bar{f}+\frac{\bar{r}^{2}}{a_{0}^{2}} \frac{\sin \bar{f}}{1-e_{0}^{2}}=\int\left(2 \frac{\bar{r}}{a_{0}} \cos \bar{f}+3 e_{0}\right) d l \\
& \frac{d}{d l} \frac{\bar{r}}{a_{0}} \cos \bar{f}=-\frac{\sin \bar{f}}{\sqrt{1-e_{0}^{2}}},
\end{aligned}
$$




$$
\frac{d}{d l} \frac{\vec{r}}{a_{0}} \sin \vec{f}=\frac{\cos \vec{f}+e_{0}}{\sqrt{1-e_{0}^{2}}},
$$

we deduce from Equation 16:

$$
\begin{aligned}
\frac{d \Psi}{d t}= & -n_{0} y\left(\Upsilon+2 \frac{h}{h_{0}} \frac{e_{0}}{1-e_{0}^{2}}\right) \\
& +M_{2} \frac{\partial \Omega}{\partial l}+N_{2} \cdot r \frac{\partial \Omega}{\partial r}
\end{aligned}
$$

where

$$
\begin{aligned}
& M_{2}=\frac{2 a_{0} n_{0}}{1-e_{0}^{2}}\left[\frac{1}{\sqrt{1-e_{0}^{2}}} \int\left(2 \frac{\bar{r}}{a_{0}} \cos \overline{\mathrm{f}}+3 e_{0}\right) d l-\frac{\nu}{1+\nu} \cdot \frac{\bar{r}}{a_{0}} \sin \bar{f}+\left(\frac{h^{2}}{h_{0}^{2}}-1\right) \cdot \frac{\bar{r}^{2}}{a_{0}^{2}} \cdot \frac{\sin \bar{f}}{1-e_{0}^{2}}\right] \\
& \begin{aligned}
N_{2}= & \frac{2 a_{0} n_{0}}{\left(1-e_{0}^{2}\right)^{3 / 2}}\left[-\left(\frac{\bar{r}}{a_{0}} \cos \overline{\mathrm{f}}+2 e_{0}\right)+\sqrt{1-e_{0}^{2}} \cdot \frac{\nu}{1+\nu} \frac{d}{d l} \frac{\bar{r}}{a_{0}} \sin \bar{f}\right. \\
& \left.+\left(\frac{h^{2}}{h_{0}^{2}}-1\right) e_{0} \frac{\bar{r}}{a_{0}} \frac{\sin \bar{f}}{\sqrt{1-e_{0}^{2}} \frac{d}{d l}} \frac{\bar{r}}{a_{0}} \cos \bar{f}\right] .
\end{aligned}
\end{aligned}
$$

Equation 14 takes a very simple form:

$$
\begin{gathered}
\frac{\mathrm{d}}{\mathrm{dt}} \frac{\mathrm{h}_{0}}{\mathrm{~h}}=\mathrm{M}_{3} \frac{\partial \Omega}{\partial l}+\mathrm{N}_{3} \cdot \mathrm{r} \frac{\partial \Omega}{\partial \mathrm{r}}, \\
M_{3}=\frac{\mathrm{n}_{0} \mathrm{a}_{0}}{1-\mathrm{e}_{0}{ }^{2}} \frac{\overline{\mathrm{r}}^{2}}{\mathrm{a}_{0}{ }^{2}}, \\
\mathrm{~N}_{3}=-\frac{\mathrm{n}_{0} \mathrm{a}_{0}}{1-\mathrm{e}_{0}{ }^{2}} \cdot \frac{\overline{\mathrm{r}}}{\mathrm{a}_{0}} \cdot \frac{\mathrm{e}_{0} \sin \overline{\mathrm{f}}}{\sqrt{1-\mathrm{e}_{0}^{2}}} .
\end{gathered}
$$

Equations 18, 21, and 24 bear more resemblance to the equations from Hansen's planetary theory than to the corresponding equations from his lunar theory, and they can be used to serve as a basis of a purely numerical development. The value of $n_{0} y$ must be determined in such a way that no purely secular term is present in Equation 21. 


\section{PERTURBATIONS OF THE POSITION OF THE ORBIT PLANE}

In previous work (Reference 4) the author has established the following equations for the determination of $\lambda$ parameters:

$$
\begin{aligned}
& \frac{d \lambda_{1}}{d t}=+n_{0} a \lambda_{2}+\frac{1}{2} h r \frac{\partial \Omega}{\partial Z}\left[+\lambda_{4} \cos (\bar{f}+\omega)-\lambda_{3} \sin (\bar{f}+\omega)\right], \\
& \frac{d \lambda_{2}}{d t}=-n_{0} a \lambda_{1}+\frac{1}{2} h r \frac{\partial \Omega}{\partial Z}\left[-\lambda_{3} \cos (\bar{f}+\omega)-\lambda_{4} \sin (\bar{f}+\omega)\right] \\
& \frac{d \lambda_{3}}{d t}=+n_{0} \eta \lambda_{4}+\frac{1}{2} h r \frac{\partial \Omega}{\partial Z}\left[+\lambda_{2} \cos (\bar{f}+\omega)+\lambda_{1} \sin (\bar{f}+\omega)\right], \\
& \frac{d \lambda_{4}}{d t}=-n_{0} \eta \lambda_{3}+\frac{1}{2} h r \frac{\partial \Omega}{\partial Z}\left[-\lambda_{1} \cos (\bar{f}+\omega)+\lambda_{2} \sin (\bar{f}+\omega)\right] .
\end{aligned}
$$

We have

$$
\frac{\partial \Omega}{\partial Z}=-m^{\prime}\left(\frac{1}{\Delta^{3}}-\frac{1}{r^{\prime 3}}\right) r^{\prime} \sin \left(v^{\prime}-\psi^{\prime}\right) \sin I .
$$

Taking the relations

$$
v^{\prime}=\bar{f}^{\prime}+\pi_{0}^{\prime}+n_{0} y^{\prime} t
$$

and

$$
\psi^{\prime}=\psi_{0}^{\prime}-n_{0}(\alpha+\eta) t+K-N
$$

into account, we obtain from Equation 31:

$$
\frac{1}{2} \frac{\partial \Omega}{\partial \bar{Z}}=- \text { in }^{\prime}\left(\frac{1}{\Delta^{3}}-\frac{1}{r^{\prime 3}}\right) r^{\prime}\left[\left(\lambda_{2} \lambda_{4}-\lambda_{1} \lambda_{3}\right) \cos \left(\bar{f}^{\prime}+\omega^{\prime}\right)+\left(\lambda_{1} \lambda_{4}+\lambda_{2} \lambda_{3}\right) \sin \left(\bar{f}^{\prime}+\omega^{\prime}\right)\right] .
$$

We have from Equation 7:

$$
\begin{aligned}
& \frac{1}{2} \frac{\partial S}{\partial \lambda_{1}}=+\lambda_{1} \cos \left(\bar{f}+\bar{f}^{\prime}+\omega+\omega^{\prime}\right)-\lambda_{2} \sin \left(\bar{f}+\bar{f}^{\prime}+\omega+\omega^{\prime}\right), \\
& \frac{1}{2} \frac{\partial S}{\partial \lambda_{2}}=-\lambda_{2} \cos \left(\bar{f}+\bar{f}^{\prime}+\omega+\omega^{\prime}\right)-\lambda_{1} \sin \left(\bar{f}+\bar{f}^{\prime}+\omega+\omega^{\prime}\right),
\end{aligned}
$$




$$
\begin{aligned}
& \frac{1}{2} \frac{\partial S}{\partial \lambda_{3}}=-\lambda_{3} \cos \left(\overline{\mathrm{f}}-\overline{\mathrm{f}}^{\prime}+\omega-\omega^{\prime}\right)-\lambda_{4} \sin \left(\overline{\mathrm{f}}-\overline{\mathrm{f}}^{\prime}+\omega-\omega^{\prime}\right), \\
& \frac{1}{2} \frac{\partial \mathrm{S}}{\partial \lambda_{4}}=+\lambda_{4} \cos \left(\overline{\mathrm{f}}-\overline{\mathrm{f}}^{\prime}+\omega-\omega^{\prime}\right)-\lambda_{3} \sin \left(\overline{\mathrm{f}}-\overline{\mathrm{f}}^{\prime}+\omega-\omega^{\prime}\right) .
\end{aligned}
$$

Substituting Equation 32 into Equations 27 to 30 and taking Equations 33 to 36 into account, we deduce the system of equations that replace Hansen's equations for his $P, Q$, and $K$ parameters:

$$
\begin{aligned}
& \frac{d \lambda_{1}}{d t}=+n_{0} a \lambda_{2}+\frac{1}{4} \frac{h}{h_{0}} \cdot \frac{a_{0} n_{0}}{\sqrt{1-e_{0}^{2}}}\left[+\left(\lambda_{4}{ }^{2}+\lambda_{3}{ }^{2}\right) \frac{\partial \Omega}{\partial \lambda_{2}}-\left(\lambda_{1} \lambda_{4}+\lambda_{2} \lambda_{3}\right) \frac{\partial \Omega}{\partial \lambda_{3}}-\left(\lambda_{2} \lambda_{4}-\lambda_{1} \lambda_{3}\right) \frac{\partial \Omega}{\partial \lambda_{4}}\right],(3 \\
& \frac{d \lambda_{2}}{d t}=-n_{0} a \lambda_{1}+\frac{1}{4} \frac{h}{h_{0}} \cdot \frac{a_{0} n_{0}}{\sqrt{1-e_{0}^{2}}}\left[-\left(\lambda_{4}{ }^{2}+\lambda_{3}^{2}\right) \frac{\partial \Omega}{\partial \lambda_{1}}-\left(\lambda_{2} \lambda_{4}-\lambda_{1} \lambda_{3}\right) \frac{\partial \Omega}{\partial \lambda_{3}}+\left(\lambda_{1} \lambda_{4}+\lambda_{2} \lambda_{3}\right) \frac{\partial \Omega}{\partial \lambda_{4}}\right],(3 \\
& \frac{d \lambda_{3}}{d t}=+n_{0} \eta \lambda_{4}+\frac{1}{4} \frac{h}{h_{0}} \cdot \frac{a_{0} n_{0}}{\sqrt{1-e_{0}^{2}}}\left[-\left(\lambda_{1}^{2}+\lambda_{2}^{2}\right) \frac{\partial \Omega}{\partial \lambda_{4}}+\left(\lambda_{1} \lambda_{4}+\lambda_{2} \lambda_{3}\right) \frac{\partial \Omega}{\partial \lambda_{1}}+\left(\lambda_{2} \lambda_{4}-\lambda_{1} \lambda_{3}\right) \frac{\partial \Omega}{\partial \lambda_{2}}\right], \\
& \frac{d \lambda_{4}}{d t}=-n_{0} \eta \lambda_{3}+\frac{1}{4} \frac{i}{h_{0}} \cdot \frac{a_{0} n_{0}}{\sqrt{1-e_{0}^{2}}}\left[+\left(\lambda_{1}^{2}+\lambda_{2}^{2}\right) \frac{\partial \Omega}{\partial \lambda_{3}}+\left(\lambda_{2} \lambda_{4}-\lambda_{1} \lambda_{3}\right) \frac{\partial \Omega}{\partial \lambda_{1}}-\left(\lambda_{1} \lambda_{4}+\lambda_{2} \lambda_{3}\right) \frac{\partial \Omega}{\partial \lambda_{2}}\right] .
\end{aligned}
$$

If necessary, the effect of the secular motion of the disturbing body's orbit can be easily taken into account. We have for this effect (Reference 6):

$$
\begin{gathered}
\frac{\mathrm{dI}}{\mathrm{dt}}=-\frac{\mathrm{di}}{\mathrm{dt}} \cos \left(\psi^{\prime}-\sigma^{\prime}\right)-\frac{\mathrm{d} \Omega^{\prime}}{\mathrm{dt}} \sin \mathrm{i}^{\prime} \sin \left(\psi^{\prime}-\sigma^{\prime}\right), \\
\frac{\mathrm{dN}}{\mathrm{dt}}=+\frac{1}{2}\left[-\frac{\mathrm{di}}{\mathrm{dt}} \sin \left(\psi^{\prime}-\sigma^{\prime}\right)+\frac{\mathrm{d} \Omega^{\prime}}{\mathrm{dt}} \sin \mathrm{i}^{\prime} \cos \left(\psi^{\prime}-\sigma^{\prime}\right)\right] \operatorname{ctg} \frac{1}{2} \mathrm{I}, \\
\frac{\mathrm{dK}}{\mathrm{dt}}=+\frac{1}{2}\left[-\frac{\mathrm{di}}{\mathrm{dt}} \sin \left(\psi^{\prime}-\sigma^{\prime}\right)+\frac{\mathrm{d} \Omega^{\prime}}{\mathrm{dt}} \sin \mathrm{i}^{\prime} \cos \left(\psi^{\prime}-\sigma^{\prime}\right)\right] \operatorname{tg} \frac{1}{2} \mathrm{I} .
\end{gathered}
$$

We can neglect the effect of change of $i$ ' on the motion of the satellite. Substituting

$$
\psi^{\prime}=\psi_{0}^{\prime}-\mathrm{n}_{0}(\alpha+\eta)+\mathrm{K}-\mathrm{N}
$$

into Equations 41 to 43 and putting

$$
\theta=\left(\pi_{0}^{\prime}+n_{0} y^{\prime} t-\sigma^{\prime}\right)-\omega^{\prime},
$$


we obtain the effect of the secular motion of the disturbing body's orbit plane on the relative position of the satellite's orbit plane in the form:

$$
\begin{aligned}
& \frac{d \partial \lambda_{1}}{d t}=-\frac{1}{2}\left(+\lambda_{3} \cos \theta+\lambda_{4} \sin \theta\right) \cdot \sin i \cdot \frac{d \Omega^{\prime}}{d t}, \\
& \frac{d \partial \lambda_{2}}{d t}=+\frac{1}{2}\left(+\lambda_{4} \cos \theta-\lambda_{3} \sin \theta\right) \cdot \sin i \cdot \frac{d \Omega^{\prime}}{d t}, \\
& \frac{d \partial \lambda_{3}}{d t}=+\frac{1}{2}\left(+\lambda_{1} \cos \theta+\lambda_{2} \sin \theta\right) \cdot \sin i \cdot \frac{d \Omega^{\prime}}{d t}, \\
& \frac{d \partial \lambda_{4}}{d t}=-\frac{1}{2}\left(+\lambda_{2} \cos \theta-\lambda_{1} \sin \theta\right) \cdot \sin i \cdot \frac{d \Omega^{\prime}}{d t} .
\end{aligned}
$$

The combined effect of direct perturbations with the secular motion of the orbital plane of the disturbing body is given by the system of equations representing the sum of Equations 37 to 40 and of 44 to 47 .

$$
\begin{aligned}
& \frac{d \lambda_{1}}{d t}=+n_{0} a \lambda_{2}+\frac{1}{4} \frac{h}{i_{0}} \cdot \frac{a_{0} n_{0}}{\sqrt{1-e_{0}{ }^{2}}}\left[+\left(+\lambda_{4}{ }^{2}+\lambda_{3}{ }^{2}\right) \frac{\partial \Omega}{\partial \lambda_{2}}-\left(+\lambda_{1} \lambda_{4}+\lambda_{2} \lambda_{3}\right) \frac{\partial \Omega}{\partial \lambda_{3}}-\left(+\lambda_{2} \lambda_{4}-\lambda_{1} \lambda_{3}\right) \frac{\partial \Omega}{\partial \lambda_{4}}\right] \\
& -\frac{1}{2}\left(+\lambda_{3} \cos \theta+\lambda_{4} \sin \theta\right) \cdot \sin i \cdot \frac{d \Omega^{\prime}}{d t}, \\
& \frac{\mathrm{d} \lambda_{2}}{\mathrm{dt}}=-\mathrm{n}_{0} a \lambda_{1}+\frac{1}{4} \frac{\mathrm{h}}{h_{0}} \cdot \frac{\mathrm{a}_{0} \mathrm{n}_{0}}{\sqrt{1-\mathrm{e}_{0}{ }^{2}}}\left[-\left(\lambda_{4}{ }^{2}+\lambda_{3}{ }^{2}\right) \frac{\partial \Omega}{\partial \lambda_{1}}-\left(+\lambda_{2} \lambda_{4}-\lambda_{1} \lambda_{3}\right) \frac{\partial \Omega}{\partial \lambda_{3}}+\left(+\lambda_{1} \lambda_{4}+\lambda_{2} \lambda_{3}\right) \frac{\partial \Omega}{\partial \lambda_{4}}\right] \\
& +\frac{1}{2}\left(+\lambda_{4} \cos \theta-\lambda_{3} \sin \theta\right) \cdot \sin i \cdot \frac{d \Omega^{\prime}}{d t}, \\
& \frac{d \lambda_{3}}{d t}=+n_{0} \eta \lambda_{4}+\frac{1}{4} \frac{h}{h_{0}} \cdot \frac{a_{0} n_{0}}{\sqrt{1-e_{0}^{2}}}\left[-\left(\lambda_{1}{ }^{2}+\lambda_{2}{ }^{2}\right) \frac{\partial \Omega}{\partial \lambda_{4}}+\left(+\lambda_{1} \lambda_{4}+\lambda_{2} \lambda_{3}\right) \frac{\partial \Omega}{\partial \lambda_{1}}+\left(+\lambda_{2} \lambda_{4}-\lambda_{1} \lambda_{3}\right) \frac{\partial \Omega}{\partial \lambda_{2}}\right] \\
& +\frac{1}{2}\left(+\lambda_{1} \cos \theta+\lambda_{2} \sin \theta\right) \cdot \sin \mathrm{i}^{\prime} \frac{\mathrm{d} \Omega^{\prime}}{\mathrm{dt}} \\
& \frac{\mathrm{d} \lambda}{\mathrm{dt}}=-\mathrm{n}_{0} \eta \lambda_{3}+\frac{1}{4} \frac{\mathrm{h}}{\mathrm{h}_{0}} \cdot \frac{\mathrm{a}_{0} \mathrm{n}_{0}}{\sqrt{1-\mathrm{e}_{0}{ }^{2}}}\left[+\left(\lambda_{1}{ }^{2}+\lambda_{2}^{2}\right) \frac{\partial \Omega}{\partial \lambda_{3}}+\left(+\lambda_{2} \lambda_{4}-\lambda_{1} \lambda_{3}\right) \frac{\partial \Omega}{\partial \lambda_{1}}-\left(+\lambda_{1} \lambda_{4}+\lambda_{2} \lambda_{3}\right) \frac{\partial \Omega}{\partial \lambda_{2}}\right] \\
& -\frac{1}{2}\left(+\lambda_{2} \cos \theta-\lambda_{1} \sin \theta\right) \cdot \sin \mathrm{i}^{\prime} \frac{\mathrm{d} \Omega^{\prime}}{\mathrm{dt}}
\end{aligned}
$$

The values of $\alpha$ and $\eta$ must be determined in such a way that no terms linear in time are present in Equations 49 and 50. 


\section{DETERMINATION OF CONSTANTS OF INTEGRATION}

The series for $\Psi$ has the form

$$
\Psi=\sum \sin \left(i g+i^{\prime} g^{\prime}+j \omega+j^{\prime} \omega^{\prime}\right),
$$

and no additive constant of integration appears in this development. The secular motion $\mathrm{n}_{0} \mathrm{y}$ of the perigee of the satellite in its orbit plane is determined in such a way that no constant term is present in Equation 21 for $\mathrm{d} \Psi / \mathrm{dt}$ and, consequently, no secular term appears in Equation 52 . The series for

$$
\frac{\mathrm{h}_{0}}{\mathrm{~h}} \text { and } \mathrm{r}
$$

both will have the form

$$
\sum C \cos \left(i g+i^{\prime} g^{\prime}+j \omega+j^{\prime} \omega^{\prime}\right),
$$

and an additive constant of integration is associated with the series of the form 53 . Let

$$
\left[\frac{h_{0}}{h}\right],[\Upsilon], \text { and }[\Psi]
$$

be the series obtained by the formal integration procedure as applied to Equations 18, 21, and 24 . We then have

$$
\begin{gathered}
\frac{h_{0}}{h}=1+c_{1}+\left[\frac{h_{0}}{h}\right]=1+\Delta, \\
\Upsilon=c_{2}+[\Upsilon], \\
\Psi=[\Psi] .
\end{gathered}
$$

At this point it is convenient to introduce Hansen's $\equiv$ and w functions. We have (Hansen, Reference 1)

$$
\equiv=-1-\frac{h_{0}}{h}+2 \frac{h}{h_{0}}-\frac{3}{2} e_{0} \Upsilon \text {. }
$$

Taking Equations 54 and 55 into account, we can write the last equation in the form

$$
\bar{E}=-3 \hat{\Delta}-\frac{3}{2} \mathrm{e}_{0} r+2\left(\Delta^{2}-\Delta^{3}+\cdots\right)
$$


or in the form

$$
\equiv=\left(-3 c_{1}-\frac{3}{2} e_{0} c_{2}\right)+[\equiv]
$$

where we put

$$
\begin{aligned}
{[\Xi]=} & -3\left[\frac{h_{0}}{h}\right]-\frac{3}{2} e_{0}[\Upsilon] \\
& +2\left(\Delta^{2}-\Delta^{3}+\cdots\right) .
\end{aligned}
$$

The value for $\Delta$ in the last equation can be taken from the previous approximation.

We put with Hansen:

$$
\begin{aligned}
& \bar{W}_{0}=\Xi+\Upsilon\left(\frac{\bar{\rho}}{a_{0}} \cos \bar{\varphi}+\frac{3}{2} e_{0}\right)+\Psi \frac{\bar{\rho}}{a_{0}} \sin \bar{\varphi} \\
& \bar{W}=\Xi+\Upsilon\left(\frac{\bar{r}}{a_{0}} \cos \bar{f}+\frac{3}{2} e_{0}\right)+\Psi \frac{\bar{r}}{a_{0}} \sin \bar{f} .
\end{aligned}
$$

We have:

$$
\begin{aligned}
& \bar{W}-\bar{W}_{0}=\Upsilon\left(\frac{\bar{r}}{a_{0}} \cos \bar{f}-\frac{\bar{\rho}}{a_{0}} \cos \bar{\varphi}\right)+\Psi\left(\frac{\bar{r}}{a_{0}} \sin \bar{f}-\frac{\bar{\rho}}{a_{0}} \sin \bar{\varphi}\right), \\
& \left(\frac{\bar{r}}{a_{0}} \cos \bar{f}\right)-\left(\frac{\bar{\rho}}{a_{0}} \cos \bar{\varphi}\right)=\sum_{n} \frac{\left(n_{0} \delta z\right)^{n}}{n !} \frac{d^{n}}{d^{n}} \frac{\bar{\rho}}{a_{0}} \cos \bar{\varphi}, \\
& \left(\frac{\bar{r}}{a_{0}} \sin \bar{f}\right)-\left(\frac{\bar{\rho}}{a_{0}} \sin \bar{\varphi}\right)=\sum_{n} \frac{\left(n_{0} \delta z\right)^{n}}{n !} \frac{d^{n}}{d^{n}} \frac{\bar{\rho}}{a_{0}} \sin \bar{\varphi} ;
\end{aligned}
$$

in addition,

$$
\left(\frac{\mathrm{r}}{\mathrm{a}_{0}}\right)^{2}-\left(\frac{\bar{\rho}}{\mathrm{a}_{0}}\right)^{2}=\sum_{\mathrm{n}} \frac{\left(\mathrm{n}_{0} \delta z\right)^{\mathrm{n}}}{\mathrm{n} !} \frac{\mathrm{d}^{\mathrm{n}}}{\mathrm{dg}^{\mathrm{n}}} \frac{\bar{\rho}^{2}}{\mathrm{a}_{0}{ }^{2}} .
$$

The value of $n_{0} \delta z$ taken from the previous approximation can be used in the computation of the righthand sides of Equations 63 to 66 for our purposes. 
The generalized Hill's formula (Reference 7)

$$
\frac{\mathrm{dn}_{0} \partial z}{\mathrm{dt}}=\mathrm{n}_{0} \frac{\overline{\mathrm{W}}+z^{2}}{1-\nu^{2}}-\frac{\mathrm{n}_{0} \mathrm{y}}{\sqrt{1-\mathrm{e}_{0}^{2}}} \frac{\bar{r}^{2}}{\mathrm{a}_{0}^{2}}
$$

can be written in the form

$$
\frac{\mathrm{dn}_{0} \delta z}{\mathrm{dt}}=\mathrm{n}_{0} \bar{w}+\frac{\mathrm{n}_{0} \nu^{2}(\bar{w}+1)}{1-\nu^{2}}-\frac{\mathrm{n}_{0} \mathrm{y}}{\sqrt{1-\mathrm{e}_{0}^{2}}} \frac{\overline{\mathrm{r}}^{2}}{\mathrm{a}_{0}^{2}}
$$

or in the form

$$
\frac{d n_{0} \partial z}{d t}=n_{0} \bar{W}_{0}-\frac{n_{0} y}{\sqrt{1-e_{0}^{2}}} \frac{\bar{\rho}^{2}}{a_{0}^{2}}+B
$$

where

$$
B=n_{0}\left(\bar{W}-\bar{W}_{0}\right)-\frac{n_{0} y}{\sqrt{1-e_{0}^{2}}}\left(\frac{\bar{r}^{2}}{a_{0}^{2}}-\frac{\bar{\rho}^{2}}{a_{0}^{2}}\right)+\frac{n_{0} \nu^{2}(\bar{W}+1)}{1-\nu^{2}} .
$$

The value of $B$ is computed on the basis of the previous approximation. Designating by $\left[\bar{W}_{0}\right]$ the value of $\bar{W}_{0}$ as obtained using the formal integration procedure, we have

$$
\begin{gathered}
\bar{W}_{0}=+\left(-3 c_{1}-\frac{3}{2} e_{0} c_{2}\right)+c_{2}\left(\frac{\bar{\rho}}{a_{0}} \cos \bar{\varphi}+\frac{3}{2} e_{0}\right)+\left[\bar{W}_{0}\right], \\
{\left[\bar{W}_{0}\right]=[\Xi]+[\Upsilon]\left(\frac{\bar{\rho}}{a_{0}} \cos \bar{\varphi}+\frac{3}{2} e_{0}\right)+[\Psi] \frac{\bar{\rho}}{a_{0}} \sin \bar{\varphi} ;}
\end{gathered}
$$

and Equation 69 takes the form

$$
\frac{\mathrm{dn} n_{0} \delta \mathbf{z}}{\mathrm{dt}}=\mathrm{n}_{0}\left(-3 c_{1}-\frac{3}{2} \mathrm{e}_{0} c_{2}\right)+\mathrm{n}_{0} \mathrm{c}_{2}\left(\frac{\bar{\rho}}{\mathrm{a}_{0}} \cos \bar{\varphi}+\frac{3}{2} \mathrm{e}_{0}\right)-\frac{\mathrm{n}_{0} \mathrm{y}}{\sqrt{1-\mathrm{e}_{0}^{2}}} \frac{\bar{\rho}^{2}}{\mathrm{a}_{0}^{2}}+\mathrm{n}_{0}\left[\overline{\mathrm{W}}_{0}\right]+B \cdot
$$

Two constants of integration, $c_{1}$ and $c_{2}$, must be adjusted in such a way that no secular term and no term with the argument $g$ is present in $n_{0} \delta z$. The part

$$
\mathrm{n}_{0}\left[\overline{\mathrm{W}}_{0}\right]+\mathrm{B}-\frac{\mathrm{n}_{0} \mathrm{y}}{\sqrt{1-\mathrm{e}_{0}^{2}}} \frac{\bar{\rho}^{2}}{\mathrm{a}_{0}^{2}}
$$


contains the term of the form

$$
A_{1}+\Lambda_{2} \cos :
$$

Let $i s$ be the coefficient of the term in $\left(\bar{\rho} / a_{0}\right) \cos \bar{\phi}+(3 / 2) e_{0}$ having the argument $g$. No constant term is present in $\left(\bar{\rho} / \mathrm{a}_{0}\right) \cos \bar{\varphi}+(3 / 2) \mathrm{e}_{0}$. Thus we must have

$$
\begin{gathered}
\mathrm{n}_{0}\left(-3 c_{1}-\frac{3}{2} e_{0} c_{2}\right)+A_{1}=0, \\
n_{0} c_{2} \beta+A_{2}=0 ;
\end{gathered}
$$

and

$$
\begin{aligned}
& c_{2}=-\frac{A_{2}}{\beta n_{0}}, \\
& c_{1}=+\frac{1}{3}\left(\frac{A_{1}}{n_{0}}+\frac{3}{2} \frac{e_{0} A_{2}}{\beta n_{0}}\right) .
\end{aligned}
$$

After the constants $c_{1}$ and $c_{2}$ are determined, we obtain the perturbations of the mean anomaly by integrating Equation 69 or 73 . The perturbations of the radius vector are obtained from the formula

$$
\bar{W}=-1+2 \frac{h_{0}}{h} \cdot \frac{1}{1+\nu}-\frac{h_{0}}{h},
$$

which leads to the formula

$$
\nu=\frac{1}{2}(\Delta-\bar{W})-\frac{1}{2}(\Delta+\bar{W}) \nu,
$$

which is convenient for the use of the iteration process. This formula was used by the author in the artificial satellites theory (Reference 4).

Determination of the integration constants associated with the $\lambda$ parameters is done in exactly the same way as in Reference 4. We put

$$
\begin{gathered}
\lambda_{1}=\sin \frac{1}{2} I_{0}+\frac{1}{2}(A+B)+\left[\lambda_{1}\right], \\
\lambda_{2}=\left[\lambda_{2}\right], \\
\lambda_{3}=\left[\lambda_{3}\right],
\end{gathered}
$$




$$
\lambda_{4}=\cos \frac{1}{2} I_{0}+\frac{1}{2}(A-B)+\left[\lambda_{4}\right]
$$

where $\left[\lambda_{i}\right](i=1,2,3,4)$ are the series obtained by the formal integration procedure as applied to Equations 37 to 40 and where $A$ and $B$ are the constants of integration. We omit here the development leading to the equations for the determination of $A$ and $B$, because this development can be found in Reference 4, and give only the final results:

$$
\begin{aligned}
& A^{2}+2 A\left(\cos \frac{1}{2} I_{0}+\sin \frac{1}{2} I_{0}\right)+(11)=0, \\
& B^{2}-2 B\left(\cos \frac{1}{2} I_{0}-\sin \frac{1}{2} I_{0}\right)+(12)=0,
\end{aligned}
$$

where

$$
(11)=\text { const. part in }\left\{\left(\left[\lambda_{1}\right]+\left[\lambda_{4}\right]\right)^{2}+\left(\left[\lambda_{2}\right]-\left[\lambda_{3}\right]\right)^{2}\right\}
$$

and

$$
(12)=\text { const. part in }\left\{\left(\left[\lambda_{1}\right]-\left[\lambda_{4}\right]\right)^{2}+\left(\left[\lambda_{2}\right]+\left[\lambda_{3}\right]\right)^{2}\right\} \text {. }
$$

\section{DETERMINATION OF THE POSITION AND VELOCITY VECTORS}

We determine the position of the satellite with respect to the moving system of coordinates, whose $x$ axis is in the orbit plane of the disturbing body and is directed toward the mean position of the pericenter; the $y$ axis is in the orbit plane of the disturbing body, normal to the $x$ axis; and the $z$ axis is normal, in the positive direction, to the $x$ and $y$ axes. Introducing the matrices

$$
\begin{aligned}
& A_{1}(a)=\left[\begin{array}{ccc}
+1 & 0 & 0 \\
0 & +\cos \alpha & -\sin \alpha \\
0 & +\sin \alpha & +\cos \alpha
\end{array}\right], \\
& A_{3}(a)=\left[\begin{array}{ccc}
+\cos a & -\sin a & 0 \\
+\sin a & +\cos \alpha & 0 \\
0 & 0 & +1
\end{array}\right],
\end{aligned}
$$


we have

$$
\mathbf{r}=A_{3}\left(\psi^{\prime}-\pi_{0}^{\prime}-n_{0} y^{\prime} t\right) \cdot A_{1}(I) \cdot A_{3}\left(\pi_{0}+n_{0} y t-\psi\right)(1+\nu)\left[\begin{array}{c}
\bar{r} \cos \bar{f} \\
\bar{r} \sin \bar{f} \\
0
\end{array}\right] \text {. }
$$

\section{Substituting}

$$
\begin{aligned}
\psi & =\psi_{0}+\mathrm{n}_{0}(\eta-a)-\mathrm{K}-\mathrm{N} \\
\psi^{\prime} & =\psi_{0}^{\prime}-\mathrm{n}_{0}(\eta+a)+\mathrm{K}-\mathrm{N}
\end{aligned}
$$

into the last equation, we deduce

$$
\mathbf{r}=A_{3}\left(-\omega^{\prime}\right) \cdot \Lambda \cdot A_{3}(\omega)(1+\nu)\left[\begin{array}{c}
\bar{r} \cos \bar{f} \\
\bar{r} \sin \bar{f} \\
0
\end{array}\right] \text {, }
$$

where

$$
\begin{aligned}
\bar{r} \cos \bar{f} & =a_{0}\left(\cos E-e_{0}\right), \\
\bar{r} \sin \bar{f} & =a_{0} \sqrt{1-e_{0}^{2}} \sin E, \\
E-e_{0} \sin E & =g_{0}+n_{0} t+n_{0} \delta z ;
\end{aligned}
$$

where (Reference 4)

$$
\begin{aligned}
& \lambda_{11}=+\lambda_{1}{ }^{2}-\lambda_{2}{ }^{2}-\lambda_{3}{ }^{2}+\lambda_{4}{ }^{2}, \\
& \lambda_{12}=-2\left(\lambda_{3} \lambda_{4}+\lambda_{1} \lambda_{2}\right), \\
& \lambda_{13}=+2\left(\lambda_{1} \lambda_{3}-\lambda_{2} \lambda_{4}\right), \\
& \lambda_{21}=+2\left(\lambda_{3} \lambda_{4}-\lambda_{1} \lambda_{2}\right),
\end{aligned}
$$




$$
\begin{aligned}
& \lambda_{22}=-\lambda_{1}^{2}+\lambda_{2}^{2}-\lambda_{3}^{2}+\lambda_{4}^{2}, \\
& \lambda_{23}=-2\left(\lambda_{1} \lambda_{4}+\lambda_{2} \lambda_{3}\right), \\
& \lambda_{31}=+2\left(\lambda_{3} \lambda_{1}+\lambda_{2} \lambda_{4}\right), \\
& \lambda_{32}=+2\left(\lambda_{1} \lambda_{4}-\lambda_{2} \lambda_{3}\right), \\
& \lambda_{33}=-\lambda_{1}^{2}-\lambda_{2}^{2}+\lambda_{3}^{2}+\lambda_{4}^{2} .
\end{aligned}
$$

For the absolute velocity, but decomposed along the axes of the moving system $(x, y, z)$, we have

$$
\mathbf{v}=h A_{3}\left(\psi^{\prime}-\pi_{0}^{\prime}-n_{0} y^{\prime} t\right) \cdot A_{1}(I) \cdot A_{3}(x-\psi) \cdot\left[\begin{array}{c}
-\sin f \\
\cos f+e \\
0
\end{array}\right] \text {. }
$$

The last equation can be written in the form

$$
\mathbf{v}=h A_{3}\left(-\omega^{\prime}\right) \cdot \Lambda \cdot A_{3}(\omega) \cdot A_{3}\left(x-\pi_{0}-n_{0} y t\right) \cdot\left[\begin{array}{c}
-\sin f \\
\cos f+e \\
0
\end{array}\right] .
$$

Taking the equation

$$
f+x-\pi_{0}-n_{0} y t=\bar{f}
$$

into account, Equation 83 can be reduced to the form

$$
v=h A_{3}\left(-\omega^{\prime}\right) \cdot \Lambda \cdot A_{3}(\omega) \cdot\left[\begin{array}{c}
-\sin \bar{f}-e \sin \left(x-\pi_{0}-n_{0} y t\right) \\
+\cos \bar{f}+e \cos \left(x-\pi_{0}-n_{0} y t\right) \\
0
\end{array}\right] .
$$

Taking Equations 12 and 13 into account, we have finally:

$$
v=\frac{a_{0} n_{0}}{\sqrt{1-e_{0}^{2}}} A_{3}\left(-\omega^{\prime}\right) \cdot A \cdot A_{3}(\omega) \cdot\left[\begin{array}{cc}
-\frac{h}{h_{0}} \sin \bar{f} & -\frac{1-e_{0}^{2}}{2} \Psi \\
+\frac{h}{h_{0}}\left(\cos \bar{f}+e_{0}\right)+\frac{1-e_{0}^{2}}{2} r & 0
\end{array}\right] .
$$


Equation 85 is analogous to Equation 22 of the article published by A. Bailie and R. Bryant (Reference 8 ) on the determination of the velocity of artificial satellites. The transformation from a moving system $(x, y, z)$ to the inertial system does not represent any difficulty and therefore is omitted.

\section{COLLECTION OF FORMULAS}

Input information:

$$
\begin{aligned}
& g_{0}, w_{0}, u_{0}^{\prime}, I_{0}, a_{0}, e_{0}, n_{0}, \\
& g_{0}^{\prime}, a^{\prime}, e^{\prime}, n^{\prime}, i^{\prime}, \Omega^{\prime} .
\end{aligned}
$$

Basic arguments:

$$
\begin{aligned}
& \mathrm{g}=\mathrm{g}_{0}+\mathrm{n}_{0} \mathrm{t}, \quad \mathrm{g}^{\prime}=\mathrm{g}_{0}^{\prime}+\mathrm{n}_{0}^{\prime} \mathrm{t}, \\
& \omega=\omega_{0}+\mathrm{n}_{0}(\mathrm{y}+\mathrm{z}-\eta) \mathrm{t}, \\
& \omega^{\prime}=\omega_{0}^{\prime}+\mathrm{n}_{0}\left(a+\eta+\mathrm{y}^{\prime}\right) \mathrm{t}, \\
& \theta=\left(\pi_{0}^{\prime}+\mathrm{n}_{0} \mathrm{y}^{\prime} \mathrm{t}-\sigma^{\prime}\right)-\omega^{\prime} .
\end{aligned}
$$

We make use of the following standard formulas of the elliptic motion:

$$
\begin{aligned}
& \frac{\rho}{a_{0}} \cos \varphi=-\frac{3}{2} e_{0}+2 \sum_{p=1}^{+\infty} \frac{J_{p}^{\prime}\left(p e_{0}\right)}{p} \cos p \gamma, \\
& \frac{\rho}{a_{0}} \sin \varphi=\frac{2 \sqrt{1-e_{0}^{2}}}{e_{0}} \sum_{p=1}^{+\infty} \frac{J_{p}\left(p e_{0}\right)}{p} \sin p \gamma, \\
& \frac{\rho}{a_{0}}=1+\frac{1}{2} e_{0}^{2}-2 e_{0} \sum_{p=1}^{+\infty} \frac{J_{p}^{\prime}\left(p e_{0}\right)}{p} \cos p \gamma, \\
& \frac{\rho^{2}}{a_{0}^{2}}=1+\frac{3}{2} e_{0}^{2}-4 \sum_{p=1}^{+\infty} \frac{J_{p}\left(p e_{0}\right)}{p^{2}} \cos p \gamma, \\
& \frac{a_{0}}{\rho}=1+2 \sum_{p^{2} 1}^{+\infty} J_{p}\left(p e_{0}\right) \cos p \gamma, \\
& \frac{a^{\prime}}{r^{\prime}}=1+2 \sum_{p=1}^{+\infty} J_{p}\left(p e^{\prime}\right) \cos p g^{\prime} .
\end{aligned}
$$

If preferred, Cayley's tables can be used instead of formulas given above. 


$$
\begin{aligned}
& s_{1}=(1+v) \cdot \frac{a_{0}^{\prime}}{a_{0}} \cdot \frac{a^{\prime}}{\bar{r}} \cos \left(\varphi+\bar{f}^{\prime}+u+u^{\prime}\right) \\
& \left.s_{2}=(1+v) \cdot \frac{f}{7_{0}} \cdot \frac{a^{\prime}}{r^{\prime}} \sin \left(q+f^{\prime}+\ldots+\ldots\right)^{\prime}\right), \\
& s_{3}=(1+u) \cdot \frac{p}{a_{0}} \cdot \frac{a^{\prime}}{r^{\top}} \cdot \cos \left(p-f^{\prime}+\left(x-r^{\prime}\right),\right. \\
& s_{4}-(1+\nu) \cdot \frac{p}{\gamma_{0}} \cdot \frac{a^{\prime}}{\bar{r}^{\prime}} \cdot \sin \left(\varphi-\bar{f}^{\prime}+\omega^{\prime}-\omega^{\prime}\right), \\
& \mathrm{s}-+\left(\lambda_{1}^{2}-\lambda_{2}^{2}\right) \mathrm{s}_{1}-2 \lambda_{1} \lambda_{2} s_{2}+\left(\lambda_{4}^{2}-\lambda_{3}^{2}\right) s_{3}-2 \lambda_{3} \lambda_{4} s_{4} ; \\
& P=(1+\nu) \frac{\rho}{a_{0}} \cdot \frac{a^{\prime}}{\vec{r}} ; \\
& \frac{1}{2} \frac{\partial s}{\partial \lambda_{1}}=+\lambda_{1} s_{1}-\lambda_{2} s_{2}=\sigma_{1}, \\
& \frac{1}{2} \frac{\partial s}{\partial \lambda_{2}}=-\lambda_{2} s_{1}-\lambda_{1} s_{2}=\sigma_{2}, \\
& \frac{1}{2} \frac{\partial s}{\partial \lambda_{3}}=-\lambda_{3} s_{3}-\lambda_{4} s_{4}=\sigma_{3}, \\
& \frac{1}{2} \frac{\partial s}{\partial \lambda_{4}}=+\lambda_{4} s_{3}-\lambda_{3} s_{4}=\sigma_{4} ; \\
& M_{1}=\frac{2 a_{0} n_{0}}{1-e_{0}^{2}}\left[\frac{1}{e_{0}}\left(1-e_{0}^{2}-\frac{\rho^{2}}{a_{0}^{2}}\right)-\frac{\nu}{1+\nu} \frac{1}{e_{0}}\left(1-e_{0}^{2}-\frac{\rho}{a_{0}}\right)+\left(\frac{h^{2}}{h_{0}^{2}}-1\right) \frac{1}{e_{0}} \frac{\rho}{a_{0}}\left(1-\frac{\rho}{a_{0}}\right)\right], \\
& N_{1}=\frac{2 a_{0} n_{0}}{1-\mathrm{e}_{0}^{2}} \cdot \frac{\rho}{a_{0}} \cdot \frac{\sin \varphi}{\sqrt{1-\mathrm{e}_{0}^{2}}}\left[1-\frac{\mathrm{a}_{0}}{\rho} \cdot \frac{\nu}{1+\nu}-\left(\frac{\mathrm{h}^{2}}{\mathrm{~h}_{0}^{2}}-1\right)\left(\frac{\mathrm{a}_{0}}{\rho}-1\right)\right], \\
& M_{2}=\frac{2 a_{0} n_{0}}{1-\mathrm{e}_{0}^{2}}\left[\frac{1}{\sqrt{1-\mathrm{e}_{0}^{2}}} \int\left(2 \frac{\rho}{\mathrm{a}_{0}} \cos \varphi+3 \mathrm{e}_{0}\right) \mathrm{d} \gamma-\frac{\nu}{1+\nu} \frac{\rho}{\mathrm{a}_{0}} \sin \varphi+\left(\frac{\mathrm{h}^{2}}{\mathrm{~h}_{0}^{2}}-1\right) \frac{\rho^{2}}{\mathrm{a}_{0}^{2}} \cdot \frac{\sin \varphi}{1-\mathrm{e}_{0}^{2}}\right], \\
& N_{2}=\frac{2 a_{0} n_{0}}{\left(1-e_{0}^{2}\right)^{3 / 2}}\left[-\left(\frac{\rho}{a_{0}} \cos \varphi+2 e_{0}\right)+\sqrt{1-e_{0}^{2}} \frac{\nu}{1+2} \frac{d}{d \gamma} \frac{\rho}{a_{0}} \sin \varphi\right. \\
& \left.+\left(\frac{h^{2}}{h_{0}^{2}}-1\right) e_{0} \frac{\rho}{a_{0}} \frac{\sin \varphi}{\sqrt{1-e_{0}^{2}}} \frac{d}{d y} \frac{\rho}{a_{0}} \cos \varphi\right] \text {, } \\
& M_{3}=+\frac{n_{0} a_{0}}{1-e_{0}^{2}} \frac{\rho^{2}}{a_{0}^{2}} \text {, }
\end{aligned}
$$




$$
\begin{aligned}
& \mathrm{N}_{3}=-\frac{\mathrm{n}_{0} \mathrm{a}_{0}}{1-\mathrm{e}_{0}^{2}} \cdot \frac{\rho}{\mathrm{a}_{0}} \cdot \frac{\mathrm{e}_{0} \sin \varphi}{\sqrt{1-\mathrm{e}_{0}^{2}}} \\
& \Omega_{1}=\frac{m^{\prime} a^{2}}{a^{\prime 3}} \cdot \frac{a^{\prime}}{r^{\prime}} \cdot\left(\frac{3}{2} s^{2}-\frac{1}{2} p^{2}\right) . \\
& \Omega_{2}=\frac{m^{\prime} a^{3}}{a^{\prime 4}} \cdot \frac{a^{\prime}}{r^{\top}} \cdot\left(\frac{5}{2} s^{3}-\frac{3}{2} s p^{2}\right), \\
& \Omega_{3}=\frac{m^{\prime} a^{4}}{a^{\prime 5}} \cdot \frac{a^{\prime}}{r^{\prime}} \cdot\left(\frac{35}{8} s^{4}-\frac{15}{4} s^{2} p^{2}+\frac{3}{8} p^{4}\right), \\
& \Omega=\Omega_{1}+\Omega_{2}+\Omega_{3}+\cdots, \\
& \frac{\partial \Omega}{\partial \gamma}=\frac{\partial \Omega_{1}}{\partial \gamma}+\frac{\partial \Omega_{2}}{\partial \gamma}+\frac{\partial \Omega_{3}}{\partial \gamma}+\cdots \\
& \rho \frac{\partial \Omega}{\partial \rho}=2 \Omega_{1}+3 \Omega_{2}+4 \Omega_{3}+\cdots \\
& \frac{a}{r} \cdot \frac{r^{\prime}}{a^{\prime}} \cdot \frac{\partial \Omega}{\partial S}=+\frac{m^{\prime} a^{2}}{a^{\prime 3}} \cdot \frac{a^{\prime}}{r^{\prime}} \cdot 3 s \\
& +\frac{m^{\prime} a^{3}}{a^{\prime 4}} \cdot \frac{a^{\prime}}{r^{\prime}} \cdot\left(\frac{15}{2} s^{2}-\frac{3}{2} p^{2}\right) \\
& +\frac{m^{\prime} a^{4}}{a^{\prime} 5} \cdot \frac{a^{\prime}}{r^{\prime}} \cdot\left(\frac{35}{2} s^{3}-\frac{15}{2} s p^{2}\right)+\cdots, \\
& \mathrm{T}_{\mathrm{i}}=\mathrm{M}_{\mathrm{i}} \frac{\partial \Omega}{\partial \gamma}+\mathrm{N}_{\mathrm{i}} \cdot \rho \frac{\partial \Omega}{\partial \rho} \quad(\mathrm{i}=1,2,3) ; \\
& F_{i}=\sum_{n} \frac{1}{n !} \frac{\overline{\partial^{n} T_{i}}}{\partial \gamma^{n}}\left(n_{0} \delta z\right)^{n} \quad(n=0,1,2,3, \cdots) \\
& \frac{d \Upsilon}{d t}=+n_{0} y \Psi+F_{1} \\
& \frac{d \Psi}{d t}=-n_{0} y\left(r+2 \frac{h}{h_{0}} \cdot \frac{e_{0}}{1-e_{0}^{2}}\right)+F_{2}, \\
& \frac{d}{d t} \frac{h_{0}}{h}=F_{3}
\end{aligned}
$$




$$
\begin{aligned}
& G_{1}=\frac{1}{2} \frac{h}{h_{0}} \cdot \frac{a_{0} n_{0}}{\sqrt{1-e_{0}^{2}}} \cdot\left(\frac{a}{\rho} \cdot \frac{\overline{\mathrm{r}}^{\prime}}{a^{r}} \cdot \frac{1}{1+\nu} \frac{\partial \Omega}{\partial S}\right) \cdot\left[+\left(\lambda_{3}{ }^{2}+\lambda_{4}{ }^{2}\right) \sigma_{2}\right. \\
& -\left(\lambda_{1} \lambda_{4}+\lambda_{2} \lambda_{3}\right) \sigma_{3} \\
& \left.-\left(\lambda_{2} \lambda_{4}-\lambda_{1} \lambda_{3}\right) \sigma_{4}\right] \text {, } \\
& \mathrm{G}_{2}=\frac{1}{2} \frac{\mathrm{h}}{\mathrm{h}_{0}} \cdot \frac{\mathrm{a}_{0} \mathrm{n}_{0}}{\sqrt{1-\mathrm{e}_{0}^{2}}} \cdot\left(\frac{\mathrm{a}}{\rho} \cdot \frac{\overline{\mathrm{r}}^{\prime}}{\mathrm{a}^{\prime}} \cdot \frac{1}{1+\nu} \frac{\partial \Omega}{\partial \mathrm{S}}\right)\left[-\left(\lambda_{4}{ }^{2}+\lambda_{3}{ }^{2}\right) \sigma_{1}\right. \\
& -\left(\lambda_{2} \lambda_{4}-\lambda_{1} \lambda_{3}\right) \sigma_{3} \\
& \left.+\left(\lambda_{1} \lambda_{4}+\lambda_{2} \lambda_{3}\right) \sigma_{4}\right] \\
& G_{3}=\frac{1}{2} \frac{h}{h_{0}} \cdot \frac{a_{0} n_{0}}{\sqrt{1-e_{0}^{2}}} \cdot\left(\frac{a}{\rho} \cdot \frac{\bar{r}^{\prime}}{a} \cdot \frac{1}{1+\nu} \frac{\partial \Omega}{\partial S}\right)\left[-\left(\lambda_{1}{ }^{2}+\lambda_{2}{ }^{2}\right) \sigma_{4}\right. \\
& +\left(\lambda_{1} \lambda_{4}+\lambda_{2} \lambda_{3}\right) \sigma_{1} \\
& \left.+\left(\lambda_{2} \lambda_{4}-\lambda_{1} \lambda_{3}\right) \sigma_{2}\right] \\
& G_{4}=\frac{1}{2} \frac{h}{h_{0}} \cdot \frac{a_{0} n_{0}}{\sqrt{1-e_{0}^{2}}} \cdot\left(\frac{a}{\rho} \cdot \frac{\bar{r}^{\prime}}{a^{\prime}} \cdot \frac{1}{1+\nu} \frac{\partial \Omega}{\partial S}\right) \cdot\left[+\left(\lambda_{1}^{2}+\lambda_{2}^{2}\right) \sigma_{3}\right. \\
& +\left(\lambda_{2} \lambda_{4}-\lambda_{1} \lambda_{3}\right) \sigma_{1} \\
& \left.-\left(\lambda_{1} \lambda_{4}+\lambda_{2} \lambda_{3}\right) \sigma_{2}\right] \\
& \mathrm{H}_{\mathrm{i}}=\sum_{n} \frac{1}{\mathrm{n} !}\left(\mathrm{n}_{0} \delta \mathrm{z}\right)^{\mathrm{n}} \frac{\overline{\partial^{\mathrm{n}} \mathrm{G}_{\mathrm{i}}}}{\partial \gamma^{\mathrm{n}}} \quad(\mathrm{i}=0,1,2,3,4), \\
& \frac{d \lambda_{1}}{d t}=+n_{0} \alpha \lambda_{2}+H_{1}-\frac{1}{2}\left(+\lambda_{4} \sin \theta+\lambda_{3} \cos \theta\right) \cdot \sin i \cdot \frac{d \Omega^{\prime}}{d t} . \\
& \frac{\mathrm{d} \lambda_{2}}{\mathrm{dt}}=-\mathrm{n}_{0} a \lambda_{1}+\mathrm{H}_{2}+\frac{1}{2}\left(+\lambda_{4} \cos \theta-\lambda_{3} \sin \theta\right) \cdot \sin \mathrm{i}^{\prime} \frac{\mathrm{d} \Omega^{\prime}}{\mathrm{dt}}, \\
& \frac{\mathrm{d} \lambda_{3}}{\mathrm{dt}}=+\mathrm{n}_{0} \eta \lambda_{4}+H_{3}+\frac{1}{2}\left(+\lambda_{1} \cos \theta+\lambda_{2} \sin \theta\right) \cdot \sin \mathrm{i}^{\prime} \frac{\mathrm{d} \Omega^{\prime}}{\mathrm{dt}}, \\
& \frac{\mathrm{d} \lambda_{4}}{\mathrm{dt}}=-\mathrm{n}_{0} \lambda \lambda_{3}+\mathrm{H}_{4}+\frac{1}{2}\left(+\lambda_{1} \sin \theta-\lambda_{2} \cos \theta\right) \cdot \sin \mathrm{i}^{\prime} \cdot \frac{\mathrm{d} \Omega^{\prime}}{\mathrm{d} t} . \\
& {\left[\frac{h_{0}}{h}\right],[\Upsilon],[\Psi],\left[\lambda_{i}\right] \quad(i=1,2,3,4)}
\end{aligned}
$$

Let

be the series obtained by the formal integration process. 


$$
\begin{aligned}
& \frac{h_{0}}{h}=1+c_{1}+\left[\frac{h_{0}}{h}\right]=1+\Delta . \\
& \Upsilon=c_{2}+[\Upsilon] \text {. } \\
& \Psi=[\Psi], \\
& {[\Xi]=-3\left[\frac{h_{0}}{h}\right]-\frac{3}{2} e_{0}[\Upsilon]+2\left(\Delta^{2}-\Delta^{3}+\cdots\right),} \\
& \equiv=-3 \Delta-\frac{3}{2} e_{0} \Upsilon+2\left(\Delta^{2}-\Delta^{3}+\cdots\right) \\
& \left(\frac{\bar{r}}{a_{0}} \cos \bar{f}\right)-\left(\frac{\bar{\rho}}{a_{0}} \cos \bar{\varphi}\right)=\sum_{n} \frac{\left(n_{0} \delta z\right)^{n}}{n !} \frac{d^{n}}{d g^{n}} \frac{\bar{\rho}}{a_{0}} \cos \bar{\varphi}, \\
& \left(\frac{\bar{r}}{a_{0}} \sin \bar{f}\right)-\left(\frac{\bar{\rho}}{a_{0}} \sin \bar{\varphi}\right)=\sum \frac{\left(n_{0} \delta z\right)^{n}}{n !} \frac{d^{n}}{d^{n}} \frac{\bar{\rho}}{a_{0}} \sin \bar{\varphi}, \\
& \left(\frac{\bar{r}}{a_{0}}\right)^{2}-\left(\frac{\bar{\rho}}{a_{0}}\right)^{2}=\sum_{n} \frac{\left(n_{0} \delta z\right)^{n}}{n !} \frac{d^{n}}{d^{n}} \frac{\bar{\rho}^{2}}{a_{0}^{2}} \\
& {\left[\overline{\mathbf{W}}_{0}\right]=[\equiv]+[\Upsilon]\left(\frac{\bar{\rho}}{\mathrm{a}_{0}} \cos \bar{\varphi}+\frac{3}{2} \mathrm{e}_{0}\right)+[\Psi] \frac{\bar{\rho}}{\mathrm{a}_{0}} \sin \bar{\varphi} ;} \\
& B=n_{0}[\Upsilon]\left(\frac{\bar{r}}{a_{0}} \cos \bar{f}-\frac{\bar{\rho}}{a_{0}} \cos \bar{\varphi}\right)+[\Psi]\left(\frac{\bar{r}}{a_{0}} \sin \bar{f}-\frac{\bar{p}}{a_{0}} \sin \bar{\varphi}\right) \\
& -\frac{\mathrm{n}_{0} \mathrm{y}}{\sqrt{1-\mathrm{e}_{0}^{2}}}\left(\frac{\overline{\mathrm{r}}^{2}}{\mathrm{a}_{0}^{2}}-\frac{\bar{\rho}^{2}}{\mathrm{a}_{0}^{2}}\right)+\frac{\mathrm{n}_{0} \nu^{2}(1+\overline{\mathrm{W}})}{1-\nu^{2}} \\
& \frac{\mathrm{dn}_{0} \delta \mathrm{z}}{\mathrm{dt}}=\mathrm{n}_{0}\left(-3 \mathrm{c}_{1}-\frac{3}{2} \mathrm{e}_{0} \mathrm{c}_{2}\right)+\mathrm{n}_{0} \mathrm{c}_{2}\left(\frac{\bar{\rho}}{\mathrm{a}_{0}} \cos \bar{\varphi}+\frac{3}{2} \mathrm{e}_{0}\right) \\
& -\frac{\mathrm{n}_{0} \mathrm{y}}{\sqrt{1-\mathrm{e}_{0}^{2}}} \frac{\bar{\rho}^{2}}{\mathrm{a}_{0}^{2}}+\mathrm{n}_{0}\left[\overline{\mathrm{W}}_{0}\right]+B \\
& \mathrm{n}_{0}\left[\overline{\mathrm{W}}_{0}\right]-\frac{\mathrm{n}_{0} \mathrm{y}}{\sqrt{1-\mathrm{e}_{0}^{2}}} \cdot \frac{\bar{\rho}^{2}}{\mathrm{a}_{0}^{2}}+3=\mathrm{A}_{1}+\mathrm{A}_{2} \cos \mathrm{g}+\cdots, \\
& \frac{\bar{\rho}}{a_{0}} \cos \bar{\varphi}+\frac{3}{2} e_{0}=\beta \cos g+\cdots ;
\end{aligned}
$$




$$
\begin{aligned}
& \mathrm{c}_{\mathrm{l}}=\frac{\mathrm{A}_{1}}{3 \mathrm{n}_{0}+\mathrm{A}_{2} \mathrm{e}_{0}}+\frac{\mathrm{n}_{0}}{\mathrm{n}_{0}} . \\
& c_{2}-\frac{A_{2}}{n_{0}} \\
& w=\equiv+\Upsilon\left(\frac{\bar{r}}{a_{0}} \cos \bar{f}+\frac{3}{2} e_{0}\right)+\Psi \frac{r}{a_{0}} \sin f, \\
& \nu=\frac{1}{2}(\lambda-\bar{W})-\frac{1}{2}(\lambda+\bar{W}) v ; \\
& (11)-\text { const. in }\left\{\left(\left[\lambda_{1}\right]+\left[\lambda_{4}\right]\right)^{2}+\left(\left[\lambda_{2}\right]-\left[\lambda_{3}\right]\right)^{2}\right\} \text {, } \\
& (12)=\text { const. in }\left\{\left(\left[\lambda_{1}\right]-\left[\lambda_{4}\right]\right)^{2}+\left(\left[\lambda_{2}\right]+\left[\lambda_{3}\right]\right)^{2}\right\} \text {, } \\
& A^{2}+2 A\left(\cos \frac{1}{2} I_{0}+\sin \frac{1}{2} I_{0}\right)+(11)=0 . \\
& \mathrm{B}^{2}-23\left(\cos \frac{1}{2} \mathrm{I}_{0}-\sin \frac{1}{2} \mathrm{I}_{0}\right)+(12)=0 \\
& \lambda_{1}=\sin \frac{1}{2} I_{0}+\frac{1}{2}(A+B)+\left[\lambda_{1}\right] \text {. } \\
& \lambda_{2}=\left[\lambda_{2}\right], \\
& \lambda_{3}=\left[\lambda_{3}\right] \text {, } \\
& \lambda_{4}=\cos \frac{1}{2} I_{0}+\frac{1}{2}(A-B)+\left[\lambda_{4}\right] ; \\
& A_{1}(a)=\left[\begin{array}{ccc}
+1 & 0 & 0 \\
0 & +\cos \alpha & -\sin \alpha \\
0 & +\sin \alpha & +\cos \alpha
\end{array}\right] \\
& A_{3}(\alpha)=\left[\begin{array}{ccc}
+\cos \alpha & -\sin \alpha & 0 \\
+\sin \alpha & +\cos a & 0 \\
0 & 0 & +1
\end{array}\right] \text {, } \\
& \mathbf{r}=A_{3}\left(-\omega^{\prime}\right) \cdot \Lambda \cdot A_{3}(\omega)(1+\nu)\left[\begin{array}{c}
\bar{r} \cos \bar{f} \\
\bar{r} \sin \bar{f} \\
0
\end{array}\right] \text {, }
\end{aligned}
$$




$$
\begin{aligned}
& \mathbf{v}=\frac{a_{0} n_{0}}{\sqrt{1-e_{0}^{2}}} A_{3}\left(-\omega^{\prime}\right) \cdot \Lambda \cdot A_{3}(\omega) \cdot\left[\begin{array}{cc}
-\frac{h}{h_{0}} \sin \bar{f} & -\frac{1}{2}\left(1-e_{0}{ }^{2}\right) \Psi \\
+\frac{h}{h_{0}}\left(\cos \bar{f}+e_{0}\right)+\frac{1}{2}\left(1-e_{0}{ }^{2}\right) \Upsilon & 0
\end{array}\right] \\
& \lambda_{11}=+\lambda_{1}{ }^{2}+\lambda_{2}{ }^{2}-\lambda_{3}{ }^{2}+\lambda_{4}{ }^{2}, \\
& \lambda_{12}=-2\left(\lambda_{3} \lambda_{4}+\lambda_{1} \lambda_{2}\right) \text {. } \\
& \lambda_{13}=+2\left(\lambda_{1} \lambda_{3}-\lambda_{2} \lambda_{4}\right) \text {, } \\
& \lambda_{21}=+2\left(\lambda_{3} \lambda_{4}-\lambda_{1} \lambda_{2}\right) \text {. } \\
& \lambda_{22}=-\lambda_{1}{ }^{2}+\lambda_{2}{ }^{2}-\lambda_{3}{ }^{2}+\lambda_{4}{ }^{2} \text {. } \\
& \lambda_{23}=-2\left(\lambda_{1} \lambda_{4}+\lambda_{2} \lambda_{3}\right) \text {, } \\
& \lambda_{31}=+2\left(\lambda_{1} \lambda_{3}+\lambda_{2} \lambda_{4}\right) \text {. } \\
& \lambda_{32}=+2\left(\lambda_{1} \lambda_{4}-\lambda_{2} \lambda_{3}\right), \\
& \lambda_{33}=-\lambda_{1}^{2}-\lambda_{2}^{2}+\lambda_{3}^{2}+\lambda_{4}^{2} \text {. }
\end{aligned}
$$

\section{CONCLUSION}

A systematic investigation of the orbital stability of satellites under different initial conditions can be based on the development given herein. In light of recent results concerning the stability of lunar satellites by G. Chebotarev and S. Kirpichnikov (Reference 9), which were obtained by the method of numerical integration, it would be of interest to investigate the problem using the development into periodic series. We can expect that one of the most influential long period terms in the coordinates will be the term with the period equal to the sidereal revolution of the earth around the moon. The described method will be programmed by the Theoretical Division, Goddard Space Flight Center, for computation on the IBM 7090. We have to expect that the number of terms in the development either for planetary satellites (with the exception of the moon) or for lunar satellites will not be large, and thus the computation can be done with the limited accuracy. 


\section{REFERENCES}

1. Hansen, P. A., "Fundamenta Nova Investigationis Orbitae Verae quam Luna Perlustrat," Gotha: C. Glaeser, 1838.

2. Limekhova, E. N., "Application of Delaunay's Method to the Motion of Lunar Artificial Satellites," Byulleten Institula Teoreticheskoy Astronomii 8(7):512-529, 1962 (In Russian).

3. Hansen, P. A., "Auseinandersetzung Einer Zweckmässigen Metode zur Berechnung der Absoluten Störungen der Kleinen Planeten. Erste Abhandlung; Zweite Abhandlung," Abhandlungen der Mathematisch-Physischen Classe der Königlich Sächsischen Gesellschaft der Wissenschaften 3:41-218, 1857; 4:1-148, 1859.

4. Musen, P., "Application of Hansen's Theory to the Motion of an Artificial Satellite in the Gravitational Field of the Earth," J. Geophys. Res. 64(12):2271-2279, December 1959.

5. Cayley, A., "Tables of the Developments of Functions in the Theory of Elliptic Motion," Mem. Roy. Astronom. Soc. 29:191-306, 1859.

6. Hansen, P. A., "Darlegung der Theoretischen Berechnung der in den Mondtafeln Angewandten Störungen. Erste Abhandlung," Abhandlungen der Mathematisch-Physischen Classe der Königlich Sächsischen Gesellschaft der Wissenschaften 6:91-497, 1862.

7. Hill, G. W., "Note on Hansen's General Formulae for Perturbations," Amer. J. Math. 4:256-259, 1881.

8. Bailie, A., and Bryant, R., "Osculating Elements Derived from the Modified Hansen Theory for the Motion of an Artificial Satellite," Astronom. J. 65(8):451-453, October 1960.

9. Chebotarev, G. A., and Kirpichnikov, S. N., "On the Problem of Stability of Motion of an Artificial Lunar Satellite," Byulleten Instituta Teoreticheskoy Astronomii 8(6):402-404, 1962 (In Russian). 
Appendix A

\section{NOTATIONS}

$a_{0}$ the mean semi-major axis of the satellite's orbit

$a, e, n$ the osculating elements of the satellite

$e_{0}$ the mean eccentricity of the satellite's orbit

$f$ the osculating true anomaly

$\bar{f}$ ' the true anomaly of the disturbing body

$g=g_{0}+n_{0} t \quad$ the undisturbed mean anomaly of the satellite

$\mathrm{g}_{0} \quad$ satellite's mean anomaly at the epoch

$\mathrm{h}=\frac{\text { an }}{\sqrt{1-\mathrm{e}^{2}}}$

$h_{0}=\frac{a_{0} n_{0}}{\sqrt{1-e_{0}^{2}}}$

$I_{0}$ the mean value of the mutual inclination of two orbit planes

$-2 \mathrm{~K}$ the periodic part in $\psi-\psi^{\prime}$

$L=g_{0}+n_{0} t+n_{0} \delta z$ the perturbed mean anomaly of the satellite

$m^{\prime}$ the mass of the disturbing body

$-2 \mathrm{~N}$ the periodic part in $\psi+\psi^{\prime}$

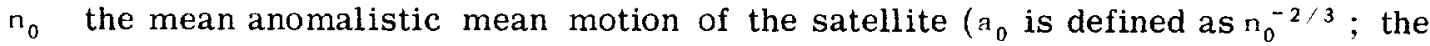
gravitational constant is put equal to 1 )

$\mathrm{n}_{0} 8 \mathrm{z}$ the perturbations in the satellite's mean anomaly

$-2 \mathrm{n}_{0}$ at the secular part in $\psi+\psi^{\prime}$

$+2 \mathrm{n}_{0} \eta \mathrm{t}$ the secular part in $\psi-\psi^{\prime}$

$\mathbf{r}$ the position vector of the satellite, $r=|\mathbf{r}|$

$\mathbf{r}^{\prime}$ the position vector of the disturbing body, $\left|\mathbf{r}^{\prime}\right|=r^{\prime}=\overrightarrow{\mathbf{r}}^{\prime}$ 
$\vec{r}, \bar{f} \quad$ the radius vector and the true anomaly depending upon $l$,

$\bar{r} \cos \bar{f}=a_{0}\left\{\cos E-e_{0}\right\}$

$\bar{r} \sin \bar{f}=a_{0} \sqrt{1-e_{0}^{2}} \sin F$

$E-e_{0} \sin E=l$

$S=\cos \left(r, r^{\prime}\right)$

$v$ the true orbital longitude of the satellite, reckoned from the departure point in the satellite's orbit plane

$v^{\prime}$ the true orbital longitude of the disturbing body, reckoned from the departure point in the body's orbit plane

$y$ the auxiliary mean anomaly; $y$ is replaced by $l$ at the later stage of computation

$\triangle \quad$ the distance between the satellite and the disturbing body

$\lambda_{1}, \lambda_{2}, \lambda_{3}, \lambda_{4}$ the parameters defining the periodic perturbations of the orbit plane,

$\lambda_{1}=\sin \frac{1}{2} \cos N, \lambda_{2}=\sin \frac{1}{2} \sin N$,

$\lambda_{3}=\cos \frac{1}{2} \sin \mathrm{K}, \lambda_{4}=\cos \frac{1}{2} \cos \mathrm{K}$

$\nu$ the perturbations in the radius vector $\mathrm{r}$,

$\mathbf{r}=(1+v) \overline{\mathbf{r}}$

$\pi_{0}+n_{0} y t \quad$ the mean orbital longitude of the pericenter of the satellite

$\pi_{0}^{\prime}+n_{0} y^{\prime} t$ the mean orbital longitude of the pericenter of the disturbing body

$\rho, \varphi$ the radius vector and the true anomaly depending upon the auxiliary mean anomaly $\gamma$,

$$
\begin{aligned}
& \rho \cos \varphi=a_{0}\left(\cos \epsilon-e_{0}\right) \\
& \rho \sin \varphi=a_{0} \sqrt{1-e_{0}^{2}} \sin \epsilon \\
& \epsilon-e_{0} \sin \epsilon=\gamma
\end{aligned}
$$

$\bar{\rho}, \bar{\varphi} \quad$ the radius vector and the true anomaly depending upon $\mathrm{g}$,

$$
\begin{aligned}
& \bar{\rho} \cos \bar{\varphi}=\mathrm{a}_{0}\left(\cos \bar{\epsilon}-\mathrm{e}_{0}\right) \\
& \bar{\rho} \sin \bar{\varphi}=\mathrm{a}_{0} \sqrt{1-\mathrm{e}_{0}^{2}} \sin \bar{\epsilon} \\
& \bar{\epsilon}-\mathrm{e}_{0} \sin \bar{\epsilon}=\mathrm{g}
\end{aligned}
$$


$\sigma^{\prime} \quad$ the true orbital longitude of the node of the disturbing body's orbit plane; the fixed ecliptic and equinox serve as a reference

$x \quad$ the true orbital longitude of the osculating pericenter of the satellite

$\psi$ the true orbital longitude of the common node, as reckoned from the departure point in the orbit plane of the satellite

$\psi^{\prime} \quad$ the true orbital longitude of the common node, as reckoned from the departure point in the orbit plane of the disturbing body

$\psi_{0}, \psi_{0}^{\prime} \quad$ the constant parts in $\psi$ and $\psi^{\prime}$

$\Omega$ the disturbing function

$\frac{\partial \Omega}{\partial r}$ the radial component of the disturbing force

$\frac{\partial \Omega}{\partial Z}$ the component of the disturbing force normal to the orbit plane 